\title{
Broadband excitation in solid-state NMR using interleaved DANTE pulse trains with $N$ pulses per rotor period
}

\author{
Xingyu Lu ${ }^{a}$, Julien Trébosc ${ }^{a}$, Olivier Lafon ${ }^{\mathrm{a}, *}$, Diego Carnevale ${ }^{\mathrm{b}}$, Simone Ulzega ${ }^{\mathrm{b}, \mathrm{c}}$, \\ Geoffrey Bodenhausen ${ }^{\text {b,d,e,f }}$, Jean-Paul Amoureux ${ }^{\mathrm{a}, \mathrm{g}, *}$ \\ ${ }^{a}$ Unit of Catalysis and Chemistry of Solids (UCCS), UMR 8181 CNRS, Lille North of France University, 59652 Villeneuve d'Ascq, France \\ ${ }^{\mathrm{b}}$ Institut des Sciences et Ingénierie Chimique (ISIC), Ecole Polytechnique Fédérale de Lausanne (EPFL), 1015 Lausanne, Switzerland \\ ${ }^{\mathrm{c}}$ Bruker BioSpin SA, Industriestrasse, Fällanden, Switzerland \\ ${ }^{\mathrm{d}}$ Département de Chimie, Ecole Normale Supérieure, 75231 Paris Cedex 05, France \\ e UMR 7203, CNRS/UPMC/ENS, Paris, France \\ ${ }^{\mathrm{f}}$ Université de Pierre et Marie Curie, Paris, France \\ ${ }^{\mathrm{g}}$ East China Normal University, Shanghai, China
}

\section{A R T I C L E I N F O}

\section{Article history:}

Received 28 June 2013

Revised 6 September 2013

Available online 19 September 2013

\section{Keywords:}

1D solid-state NMR

${ }^{14} \mathrm{~N}$

Broadband excitation

DANTE

MAS

Paramagnetic samples

\begin{abstract}
A B S T R A C T
We analyze the direct excitation of wide one-dimensional spectra of nuclei with spin $I=1 / 2$ or 1 in rotating solids submitted to pulse trains in the manner of Delays Alternating with Nutations for Tailored Excitation (DANTE), either with one short rotor-synchronized pulse of duration $\tau_{p}$ in each of $K$ rotor periods $\left(D_{1}^{K}\right)$ or with $N$ interleaved equally spaced pulses $\tau_{p}$ in each rotor period, globally also extending over $K$ rotor periods $\left(D_{N}^{K}\right)$. The excitation profile of $D_{N}^{K}$ scheme is a comb of $r f$-spikelets with $N v_{R}=N / T_{R}$ spacing from the carrier frequency, and a width of each spikelet inversely proportional to the length, $K T_{R}$, of $D_{N}^{K}$ scheme. Since the individual pulse lengths, $\tau_{p}$, are typically of a few hundreds of $\mathrm{ns}, D_{N}^{K}$ scheme can readily excite spinning sidebands families covering several $\mathrm{MHz}$, provided the $r f$ carrier frequency is close enough to the resonance frequency of one the spinning sidebands. If the difference of isotropic chemical shifts between distinct chemical sites is less than about $1.35 /\left(K T_{R}\right), D_{N}^{K}$ scheme can excite the spinning sidebands families of several sites. For nuclei with $I=1 / 2$, if the homogeneous and inhomogeneous decays of coherences during the DANTE sequence are neglected, the $K$ pulses of a $D_{1}^{K}$ train have a linearly cumulative effect, so that the total nutation angle is $\theta_{\text {tot }}=K 2 \pi v_{1} \tau_{p}$, where $v_{1}$ is the $r f$-field amplitude. This allows obtaining nearly ideal $90^{\circ}$ pulses for excitation or $180^{\circ}$ rotations for inversion and refocusing across wide MAS spectra comprising many spinning sidebands. If one uses interleaved DANTE trains $D_{N}^{K}$ with $N>1$, only spinning sidebands separated by intervals of $N v_{R}$ with respect to the carrier frequency are observed as if the effective spinning speed was $N v_{R}$. The other sidebands have vanishing intensities because of the cancellation of the $N$ contributions with opposite signs. However, the intensities of the remaining sidebands obey the same rules as in spectra obtained with $v_{R}$. With increasing $N$, the intensities of the non-vanishing sidebands increase, but the total intensity integrated over all sidebands decreases. Furthermore, the $N K$ pulses in a $D_{N}^{K}$ train do not have a simple cumulative effect and the optimal cumulated flip angle for optimal excitation, $\theta_{\text {tot }}^{\text {opt }}=N K 2 \pi v_{1} \tau_{p}$, exceeds $90^{\circ}$. Such $D_{N}^{K}$ pulse trains allow achieving efficient broadband excitation, but they are not recommended for broadband inversion or refocusing as they cannot provide proper $180^{\circ}$ rotations. Since $D_{N}^{K}$ pulse trains with $N>1$ are shorter than basic $D_{1}^{K}$ sequences, they are useful for broadband excitation in samples with rapid homogeneous or inhomogeneous decay.

For nuclei with $I=1$ (e.g., for ${ }^{14} \mathrm{~N}$ ), the response to basic $D_{1}^{K}$ pulse train is moreover affected by inhomogeneous decay due to 2nd-order quadrupole interactions, since these are not of rank 2 and therefore cannot be eliminated by spinning about the magic angle. For large quadrupole interactions, the signal decay produced by second-order quadrupole interaction can be minimized by (i) reducing the length of $D_{N}^{K}$ pulse trains using $N>1$, (ii) fast spinning, (iii) large $r$-field, and (iv) using high magnetic fields to reduce the 2nd-order quadrupole interaction.
\end{abstract}

(c) 2013 Elsevier Inc. All rights reserved.

\footnotetext{
* Corresponding authors. Address: Unit of Catalysis and Chemistry of Solids (UCCS), UMR 8181 CNRS, Lille North of France University, 59652 Villeneuve d'Ascq, France. Fax: +33 320436814 (J.-P. Amoureux).

E-mail addresses: olivier.lafon@univ-lille1.fr (O. Lafon), jean-paul.amoureux@ univ-lille1.fr (J.-P. Amoureux).
}

\section{Introduction}

In nuclear magnetic resonance (NMR) of solids, nuclear spins with quantum number $I$ are subject to several interactions, such 
as chemical shielding, scalar $(J)$ or dipolar couplings, quadrupolar interactions for $I \geqslant 1$, hyperfine interactions with unpaired electrons, or Knight shift produced by conduction electrons. In static powders, the resulting spectra are broadened by the anisotropy of these interactions which renders the frequency of the resonances depending on the orientation of the crystallites with respect to the static magnetic field, $B_{0}$. Usually, to average out these anisotropic terms, and thus to enhance the spectral resolution and the sensitivity, a rotation of the sample is performed about an axis tilted at the magic angle $\left(\beta_{R L}=54.7^{\circ}\right)$ with respect to $B_{0}$. When the spinning speed, $v_{R}$, is much faster than all anisotropic interactions, these are fully averaged to zero, so that the spectrum reveals a single narrow isotropic center-band resonance for each species (if $J=0$ ). When the spinning speed is slower, spinning sidebands are observed, which are separated by multiples of $v_{R}$ from the center band. In particular, there are numerous systems, in which the anisotropies of NMR interactions reach hundreds or even thousands of $\mathrm{kHz}$, and hence greatly exceed the fastest spinning speeds that can be achieved (currently $v_{R}=110 \mathrm{kHz}$ ) [1]. In that case, MAS powder spectra feature numerous spinning sidebands. Such wide spectra are encountered when: (i) the nuclei are close either to unpaired electrons in paramagnetic samples [2-12] or to conduction electrons in electrical conductors, (ii) the nuclei are subject to large quadrupole interactions $(I>1 / 2)$ [13-25], or (iii) their chemical shift anisotropy (CSA) is large, particularly when the static magnetic field is high [26,27].

Acquisition of such wide spectra is often challenging. Their signal-to-noise ratios $(\mathrm{S} / \mathrm{N})$ are low because the total integrated intensity is spread over a broad spectral width. Furthermore, the breadth of such spectra can exceed the detection bandwidth of the probe, which prevents their acquisition in a single experiment. Even when the spectrum does lie in the probe detection bandwidth, the radiofrequency $(r f$ ) fields delivered by usual solid-state NMR probes are often insufficient to achieve efficient and uniform excitation of wide powder patterns, in particular for nuclei with low gyromagnetic ratio. Furthermore, in the case of quadrupolar nuclei, the uniform excitation is rendered more difficult by the intricate spin dynamics in the presence of $r f$ fields and sample rotation.

However, several solutions have been proposed to acquire these ultra-wide-line NMR spectra [1]. The limited probe detection bandwidth can be circumvented by: (i) reducing the quality factor of the probe, at the expense of decreased sensitivity and/or (ii) piecewise spectral acquisition $[4,16,17,26]$. Wide spectra can be excited uniformly by short pulses, however at the expanse of a decrease in sensitivity [5]. Adiabatic pulses with controlled modulation of $r f$ field amplitude and carrier frequency have been demonstrated to increase the bandwidth of both excitation and inversion pulses in Carr-Purcell-Meiboom-Gill (CPMG) sequence under static conditions $[18,19,27]$. Under MAS, adiabatic pulses have been employed for broadband inversion [6-8], but they require strong $r f$-amplitudes and they have not yet been demonstrated for broadband excitation or refocusing.

In this context, trains of rotor-synchronized short rectangular pulses in the manner of Delays Alternating with Nutation for Tailored Excitation, DANTE [28,29], have been recently proposed as an efficient method for broadband excitation and inversion under MAS $[9,10,20,21,30]$. This technique has been demonstrated for the direct observation of spin- $1 / 2\left({ }^{1} \mathrm{H}\right.$ and $\left.{ }^{19} \mathrm{~F}\right)$ and spin- $1\left({ }^{6} \mathrm{Li}\right)$ nuclei subject to paramagnetic interactions [9-11], as well as the direct observation and indirect detection of spin-1 nuclei $\left({ }^{14} \mathrm{~N}\right)$ subject to large quadrupole interactions [20,21]. Furthermore, we have also recently introduced interleaved DANTE sequences comprising $N>1$ pulses per rotor period [9,20,21]. These interleaved DANTE schemes excite transverse magnetization within a shorter time and are thus useful for wide MAS spectra with several different species and/or submitted to large losses.
DANTE scheme is a Janus-faced method, being able to achieve both narrow- [14] and broad-band excitation for solids since its excitation profile is a comb of $r f$-spikelets with $N v_{R}$ spacing from the carrier frequency [17]. Here, we focus on the application of DANTE for broadband excitation, which is useful both for the 1D direct acquisition of wide spectra or their indirect detection via $2 \mathrm{D}$ experiments. In 1D experiments, DANTE sequences are used to manipulate families of sidebands spread over hundreds or thousands of $\mathrm{kHz}$. Such experiments can be achieved either by a single DANTE scheme or a DANTE spin echo [18] to avoid phase distortions related to probe dead time. The present article analyzes the performances of basic and interleaved DANTE sequences for the 1D broadband observation of wide spectra. Using numerical simulations, we demonstrate how the efficiency of DANTE sequences can be improved and how their selectivity to offset can be tailored in order to observe either a single family of sidebands or several families corresponding to different isotropic chemical shifts. We show how such efficiency enhancement and offset selectivity can be optimized by an educated choice of individual pulse length, $r f$ field amplitude, MAS frequency, total length of DANTE sequence, and number $N$ of pulses per rotor period. We examine in particular the $1 \mathrm{D}$ direct excitation of spin-1/2 nuclei in paramagnetic samples as well as that of spin- 1 nuclei $\left({ }^{14} \mathrm{~N}\right)$.

\section{Sequences}

A basic DANTE sequence applied to spinning samples consists of a train of rotor-synchronized pulses, with one pulse per rotor period, $T_{R}=1 / v_{R}$. This sequence, called $D_{1}^{K}$ in the following, lasts $K T_{R}$ (Fig. 1a). The pulses of the $D_{1}^{K}$ train have identical length, $\tau_{p}$, amplitude, $v_{1}$, and phase. The $D_{1}^{K}$ train leads to one comb of $r f$ spikelets, all separated from the carrier frequency by multiple of $v_{R}$. Globally, the $D_{1}^{K}$ sequence requires a priori the optimization of four parameters: $\tau_{p}, v_{1}, T_{R}$ and $K$.

A DANTE- $N$ sequence, referred to as $D_{N}^{K}$ in the following, consists of $N>1$ interleaved basic DANTE trains with $N$ equally spaced pulses per rotor period (Fig. $1 \mathrm{~b}-\mathrm{d}$ ). The length of $D_{N}^{K}$ sequence is identical to that of $D_{1}^{K}: K T_{R}$. The $N$ interleaved $D_{1}^{K}$ trains lead to $N$ overlapping combs of $r f$ spikelets, all separated from the carrier frequency by multiples of the MAS frequency. Here, we use $N D_{1}^{K}$ trains with identical $r f$ phase, amplitude, $v_{1}$, and pulse length, $\tau_{p}$. These conditions ensure constructive interferences and the excitation profile of $D_{N}^{K}$ sequence consists of a comb of $r f$ spikelets occurring at regular frequency intervals, $N v_{R}$, with respect to the carrier frequency [20]. Compared to $D_{1}^{K}$, there is an additional adjustable parameter in $D_{N}^{K}$ sequence: the $N$ value.

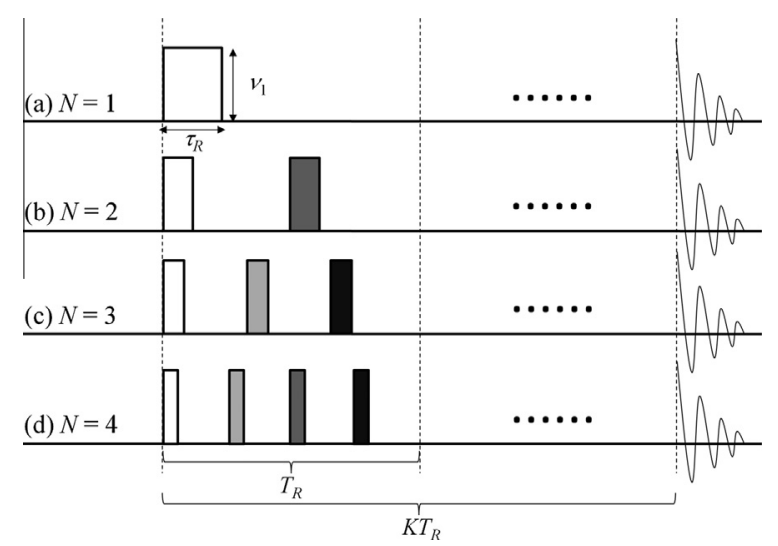

Fig. 1. Schemes for direct excitation of MAS spectra using $D_{N}^{K}$ DANTE pulse sequences with $N=1$ (a), 2 (b), 3 (c), and 4 (d). Different shades of gray correspond to the different DANTE trains. 
Another useful parameter to predict the efficiency of $D_{N}^{K}$ sequence is the total flip-angle on-resonance:

$\theta_{\text {tot }}\left({ }^{\circ}\right)=360 K N v_{1} \tau_{p}$

\section{Simulation parameters}

To rationalize the direct excitation of wide spectra by DANTE 1D methods, the spin dynamics during these pulse sequences was calculated using SIMPSON software [31]. Simulations were performed in two cases, for which direct excitation with DANTE scheme has been reported recently: ${ }^{19} \mathrm{~F}$ spin- $1 / 2$ nuclei in paramagnetic $\mathrm{CeF}_{3}$ sample [9], and ${ }^{14} \mathrm{~N}$ spin- 1 nuclei in $\gamma$-glycine $[20,21]$. The simulations have been performed with the experimental parameters for previously reported experiments: $B_{0}=18.8 \mathrm{~T}$ and $v_{R}=60$ or $62.5 \mathrm{kHz}$ for ${ }^{19} \mathrm{~F}$ and ${ }^{14} \mathrm{~N}$ direct excitation experiments, respectively.

In $\mathrm{CeF}_{3}$, the ${ }^{19} \mathrm{~F}$ nuclei are subject to hyperfine couplings with the unpaired electrons of the paramagnetic $\mathrm{Ce}^{3+}$ ions. The unpaired electron longitudinal relaxation times are several orders of magnitude shorter than those of ${ }^{19} \mathrm{~F}$ nuclei. In this 'Curie spin' limit and disregarding the anisotropy of bulk magnetic susceptibility (BMS) [32], the hyperfine couplings can be described by shifts with zeroand second-rank orientation dependences, which are formally equivalent to isotropic chemical shift and chemical shift anisotropy (CSA), respectively $[6,33,34]$. In $\mathrm{CeF}_{3}$, two distinct ${ }^{19} \mathrm{~F}$ sites have been observed, and in the simulations we have used an anisotropic de-shielding constant, $\delta_{\text {aniso }}=-814 \mathrm{ppm}$, and an asymmetry parameter, $\eta_{\mathrm{CSA}}=0.4$, which correspond to the ${ }^{19} \mathrm{~F}$ species with longest time constant $\left(T_{2}^{\prime}=1.2 \mathrm{~ms}\right)$ of transverse dephasing in spin echo experiment [9].

The ${ }^{14} \mathrm{~N}$ nuclei in $\gamma$-glycine have a quadrupolar coupling constant of $C_{Q}=1.18 \mathrm{MHz}$, and an electric field gradient (efg) asymmetry parameter of $\eta_{Q}=0.5[13,22,35]$. However, in other samples, ${ }^{14} \mathrm{~N}$ nuclei can be subject to larger quadrupole interactions [14,23,24,36]. Hence, the direct excitation using DANTE was also simulated for a single ${ }^{14} \mathrm{~N}$ site with $C_{Q}=2.36$ and $4.72 \mathrm{MHz}$ that are twice and four times as large as in $\gamma$-glycine, while preserving $\eta_{Q}=0.5$.

In all the simulations (except in Fig. 3), the carrier frequency, $v_{r}$, was set to coincide with the center-band, $v_{\text {iso }}$, resulting from: (i) isotropic chemical shift, (ii) hyperfine coupling, (iii) quadrupolar induced shift, and (iv) isotropic BMS [12]. For ${ }^{14} \mathrm{~N}$ nuclei, the second-order quadrupolar induced shift is equal to $\delta_{\text {QIS }}=-\left(C_{Q} / v_{0}\right)^{2}\left(3+\eta_{0}^{2}\right) / 32$, where $v_{0}$ is the ${ }^{14} \mathrm{~N}$ Larmor frequency $[37,38]$. In Fig. 3 , the position of carrier frequency was varied to test the robustness to offset.

The powder averages were calculated using $615\left\{\alpha_{P R}, \beta_{P R}\right\}$ pairs and 3 or $13 \gamma_{P R}$ angles. The $\left\{\alpha_{P R}, \beta_{P R}, \gamma_{P R}\right\}$ Euler angles describe the orientation of principal axes system $(\mathrm{P})$ of the interaction in the rotorfixed frame $(\mathrm{R})$. The $\left\{\alpha_{P R}, \beta_{P R}\right\}$ pairs were selected according to the REPULSION algorithm [39], whereas the $\gamma_{P R}$ angle was regularly stepped from 0 to $360^{\circ}$. Three $\gamma_{P R}$ angles were sufficient to calculate: (i) the first point of the free-induction decay (FID), which corresponds to the projection of the powder-averaged density matrix, $\langle\sigma(t)\rangle$, at the end of DANTE sequence onto the lowering operator, $I^{-}=I_{x}-i I_{y}$, (ii) the projection of $\langle\sigma(t)\rangle$ during the DANTE sequence onto the three components, $I_{x}, I_{y}$ and $I_{z}$, of nuclear spin angular momentum, and (iii) the norm of the powder averaged density matrix, $\|\langle\sigma(t)\rangle\|$. Conversely, owing to the Nyquist-Shannon sampling theorem, 13 $\gamma_{P R}$ angles were required to describe correctly the spinning sideband patterns. The projection onto the operators was calculated as

$c(Q)=(Q \mid\langle\sigma(t)\rangle)=\frac{\operatorname{Tr}\left(Q^{\dagger}\langle\sigma(t)\rangle\right)}{\operatorname{Tr}\left(Q^{\dagger} Q\right)}$,

where $Q=I_{x}, I_{y}, I_{z}$ or $I^{-}$. The FID corresponds to $c\left(I^{-}\right)$values during the acquisition and
$\|\langle\sigma(t)\rangle\|=\sqrt{c^{2}\left(I_{x}\right)+c^{2}\left(I_{y}\right)+c^{2}\left(I_{z}\right)}$,

for an isolated nucleus. The efficiency of $D_{N}^{K}$ sequence is defined as

$I I_{\text {norm }}=\frac{c\left(I^{-}, D_{N}^{K}\right)}{c\left(I^{-}, \text {ideal } 90^{\circ}\right)}$

where $c\left(I^{-}, D_{N}^{K}\right)$ and $c\left(I^{-}\right.$, ideal $\left.90^{\circ}\right)$ are the first point of FID excited either by $D_{N}^{K}$ sequence or by an ideal infinitely short $90^{\circ}$ pulse.

\section{Simulation results for ${ }^{19} \mathrm{~F}$}

\subsection{Simulation of $D_{N}^{K}{ }^{19} F 1 D$ MAS spectra}

Fig. 2 shows simulated 1D MAS NMR spectra of ${ }^{19} \mathrm{~F}$ site excited by a perfect $90^{\circ}$ pulse (Fig. $2 \mathrm{~b}$ ), a rectangular single $90^{\circ}$ pulse (Fig. 2a) and optimized $D_{N}^{14}$ sequences $(N=1-4$, Fig. 2c-f) with $v_{1}=29 \mathrm{kHz}$. The comparison of the spectra highlights that for a given $r f$ field strength, the excitation bandwidth of $D_{N}^{14}$ trains is much broader than that of a single $90^{\circ}$ pulse. These results are consistent with previously reported simulations and experiments [9]. The broadband excitation virtue of $D_{N}^{K}$ sequences stems from the use of very short pulse length (see Section 4.2). It must also be noted that spectra obtained with a perfect $90^{\circ}$ pulse and a $D_{1}^{14}$ sequence are quasi identical (compare Fig. $2 b$ and c), despite the weak $r f$-field $\left(v_{1}=29 \mathrm{kHz}\right)$ used in the second case.

The spectra of Fig. $2 \mathrm{c}-\mathrm{f}$ excited by $D_{N}^{14}$ sequences exhibit spinning sidebands spaced by $N v_{R}$ since the excitation profile of $D_{N}^{14}$ trains consists of a comb of spikelets occurring at regular frequency intervals, $\mathrm{N} v_{R}$, with respect to the carrier frequency. However, the envelopes of the $D_{N}^{14}$ spectra are similar for $N=2,3$ and 4 to that of the spectrum excited by an ideal $90^{\circ}$ pulse. In a $D_{N}^{K}$ spectrum with a full width of $F W$, the number of observable bands, $N B$, decreases with increasing $N$ value, and is approximately given by

$N B \approx 1+F W /\left(N v_{R}\right)$

$F W$ is given by $v_{0} \delta_{\text {aniso }}\left(3+\eta_{\mathrm{CSA}}\right) / 2$ for a shift anisotropy, and $3 C_{Q} / 2$ for spin-1 nucleus subject to quadrupole interaction. Hence, the spectral resolution of the powder pattern envelope decreases for increasing $N$ value and an accurate determination of CSA parameters by a fit of the $D_{N}^{K}$ spectra is not possible for too large $N$ values.

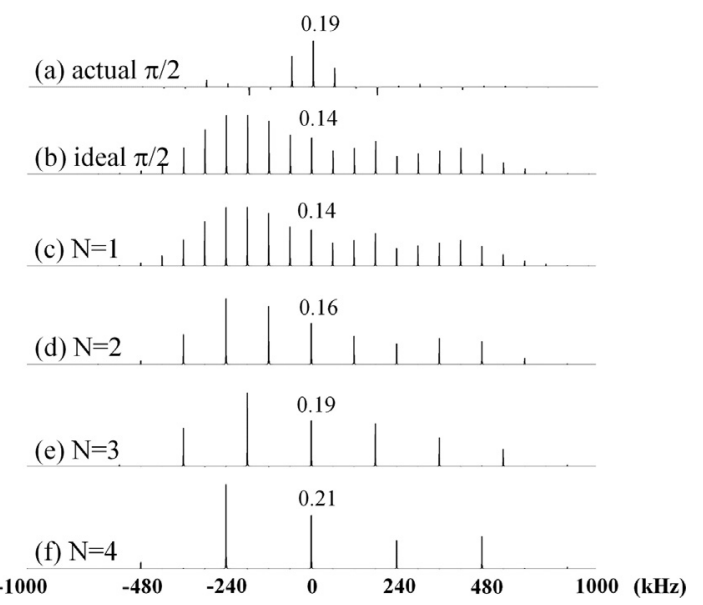

Fig. 2. Simulated 1D MAS spectra of one isolated ${ }^{19} \mathrm{~F}$ site, at $18.8 \mathrm{~T}$ with $v_{R}=60 \mathrm{kHz}$ and $v_{1}=29 \mathrm{kHz}(\mathrm{a}, \mathrm{c}-\mathrm{f})$, excited by: (a) one rectangular resonant $90^{\circ}$ pulse, (b) one ideal $90^{\circ}$ pulse, and (c-f) $D_{N}^{14}$ sequences with $N=1$ (c), 2 (d), $3(\mathrm{e})$ and 4 (f). The pulse length for each spectrum was optimized and is equal to $\tau_{p}(\mu \mathrm{s})=8.60(\mathrm{a}), 0.62$ (c), 0.38 (d), 0.31 (e), and 0.27 (f), which correspond to $\theta_{\text {tot }}=90^{\circ}, 90^{\circ}, 110^{\circ}, 135^{\circ}$ and $160^{\circ}$, respectively. These optimal conditions correspond to the crosses in Fig. 5a-d. The intensities of the center-bands are indicated in the figure. Other simulations parameters are given in Section 3. 

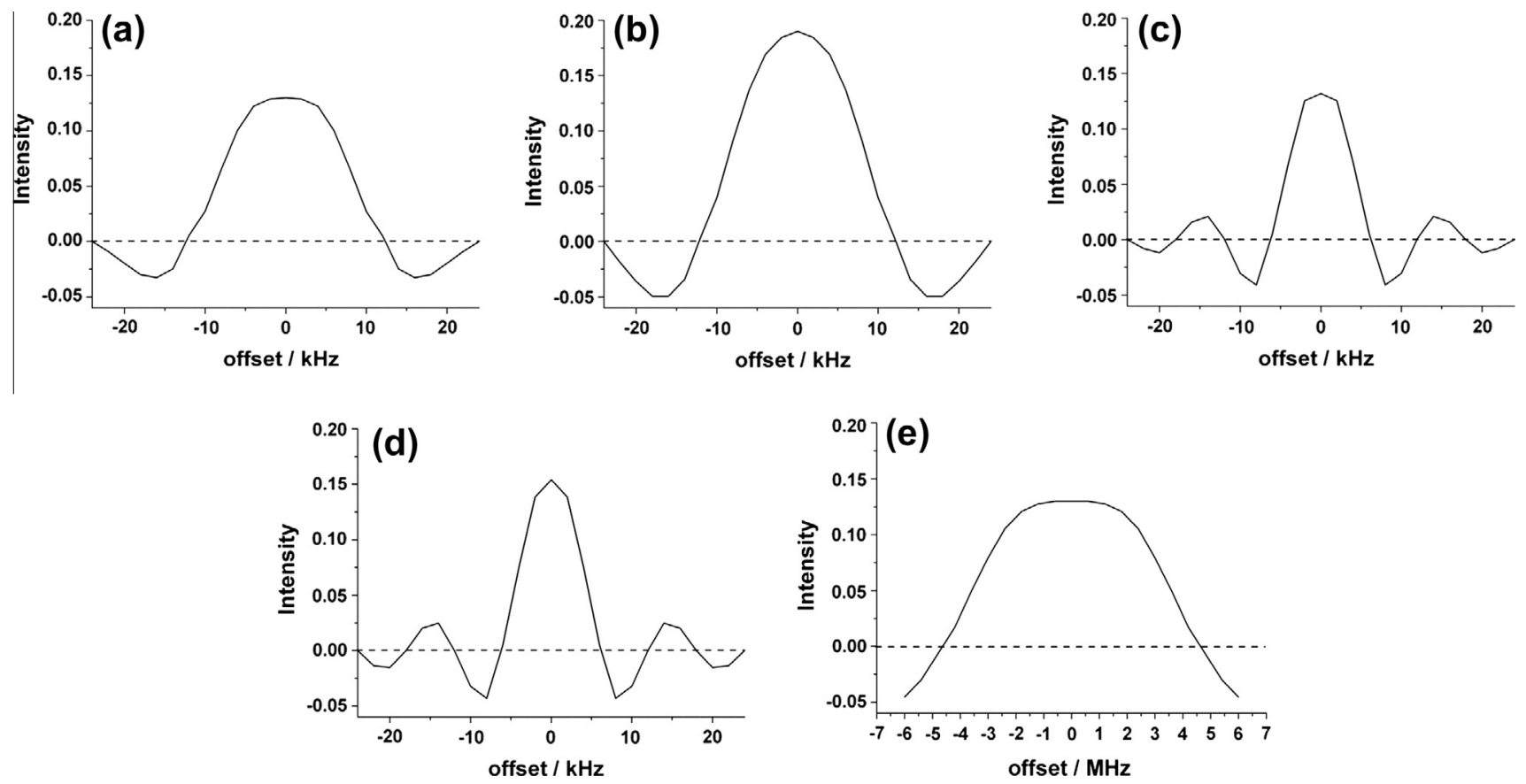

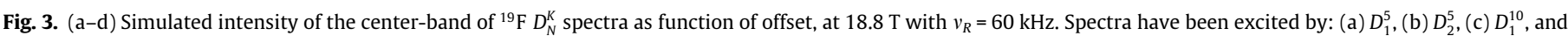

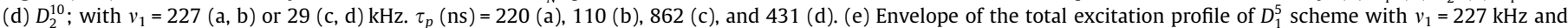
$\tau_{p}=220 \mathrm{~ns}$. It is calculated as the intensity of the center-band versus offset for $v_{r f}=v_{\text {iso }}+j v_{R}$.

\subsection{Robustness to offset and selectivity}

Fig. 3 shows the simulated excitation profile, i.e. the intensity of center-band as function of offset, for various $D_{N}^{K}$ sequences. The Fourier transform (FT) predicts that the excitation profile of $D_{N}^{K}$ scheme consists of a comb of $r f$ spikelets occurring at regular frequency intervals, $N v_{R}$, with respect to the carrier frequency and that the envelope of the comb is only determined by the FT of the individual $r f$ pulse [20]. Fig. 3a-d show the central $r f$ spikelet, when $v_{r f}$ is close to $v_{\text {iso. }}$. As predicted by FT, the $r f$ spikelets are sinc functions. Their widths do not depend on $N$ value but are inversely proportional to the total length, $K T_{R}$, of $D_{N}^{K}$ sequence. For instance, the Full-Widths of Spikelets at half-maximum $\left(F W S_{0.5}\right)$ of $D_{1}^{10}$ and $D_{2}^{10}$ sequences are identical and twofold narrower than that of $D_{1}^{5}$ and $D_{2}^{5}$ sequences. More quantitatively, we found using numerical simulations that

$F W S_{0.5} \approx 1.35 /\left(K T_{R}\right)$ and $F W S_{0.9} \approx 0.85 /\left(K T_{R}\right)$,

where $F W S_{0.9}$ is the Full-Width of Spikelets at $90 \%$ of the maximum. It must be noted that the offset-dependence behavior of the total spectrum (not shown) is identical to that of the center band shown in Fig. 3a-d. This means that spinning sidebands manifolds of species excited slightly off-resonance, but sufficiently close to the carrier frequency to be detected, are attenuated but that they remain completely similar to their values recorded on-resonance, and that they can thus be used for accurate CSA determination. According to Eq. (6), two sites with distinct center-band frequencies, $v_{\text {iso }}^{1}$ and $v_{\text {iso }}^{2}$, can be excited by a $D_{N}^{K}$ sequence when its length satisfies the relation:

$K^{\text {offset }} T_{R} \leqslant 0.85 /\left[\left|v_{\text {iso }}^{1}-v_{\text {iso }}^{2}\right|, \bmod \left(N v_{R}\right)\right]$,

In practice, the quantity $\left[\left|v_{\text {iso }}^{1}-v_{\text {iso }}^{2}\right|, \bmod \left(N v_{R}\right)\right]$ can be determined from the difference in the frequencies of closest spinning sidebands in 1D MAS spectrum excited by a single $90^{\circ}$ pulse.

Fig. 3e shows the envelope of the total excitation profile of $D_{1}^{5}$ sequence, which is also a sinc function with a width inversely proportional to $\tau_{p}$. Here, we found that Full-Widths of the Envelop are equal to:

$F W E_{0.5} \approx 1.35 / \tau_{p}$ and $F W E_{0.9} \approx 0.85 / \tau_{p}$

Usual solid-state NMR spectrometer can deliver short pulses with a length of the order of few tenths of microseconds given the duration of transients at rising and falling edges of a rectangular pulse. Note that the duration of transients is ca. inversely proportional to the Larmor frequency and hence short pulses are more difficult to achieve at low Larmor frequency, i.e. at low magnetic field and/or for nuclei with low gyromagnetic ratio. According to Eq. (8), $D_{N}^{K}$ sequences with $\tau_{p}=0.5 \mu$ s are able to fully excite MAS spectra with a breadth of $1.7 \mathrm{MHz}$ without significant distortion. More generally, undistorted MAS spectra with a full width of $F W$, can in principle be excited by a $D_{N}^{K}$ sequence with a sufficiently short pulse of

$\tau_{p} \leqslant 0.85 / F W$

For the ${ }^{19} \mathrm{~F}$ simulations reported here, $F W=1.3 \mathrm{MHz}$ and according to Eq. (9), $\tau_{p}$ must be shorter than $0.65 \mu \mathrm{s}$. Another limitation for the excitation arises from the probe bandwidth, $F W^{\text {probe }}$, which is generally proportional to the Larmor frequency. In case of broad spectra, the probe may be the limiting factor, especially at low Larmor frequency.

\subsection{Nutation curves of $D_{N}^{K}$ sequences}

Fig. 4 shows the norm of the powder-averaged density matrix and its projections onto $I_{y}$ and $I_{z}$ operators, versus $\theta_{\text {tot }}$ after a $D_{N}^{10}$ pulse train with phase $-x$, using a weak $r f$-field of $v_{1}=29 \mathrm{kHz}$. For all $D_{N}^{10}$ sequences, the projection onto $I_{x}$ operator is zero and is thus not displayed. Note that magnetization decreases produced by coherent or incoherent mechanisms, such as ${ }^{19} \mathrm{~F}-{ }^{19} \mathrm{~F}$ dipolar coupling or the BSM anisotropy, were not considered in the simulations. Experimentally, these losses reduce the magnetization for increasing $D_{N}^{K}$ length. 
(a) $\mathrm{N}=1$

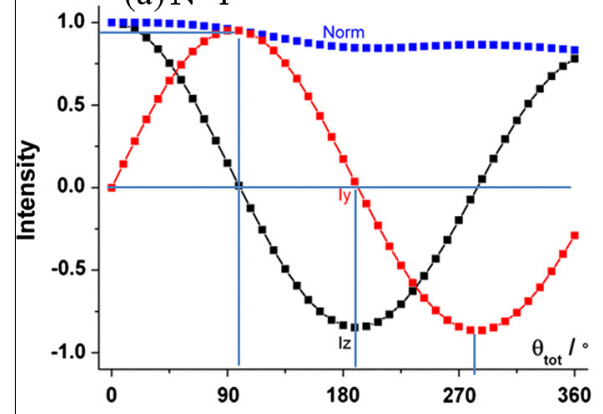

(c) $\mathrm{N}=3$

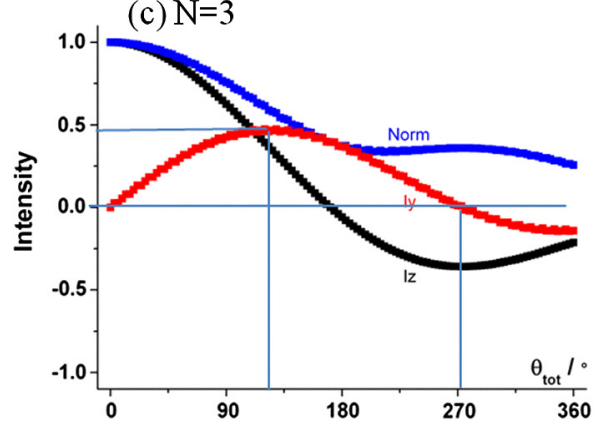

(b) $\mathrm{N}=2$

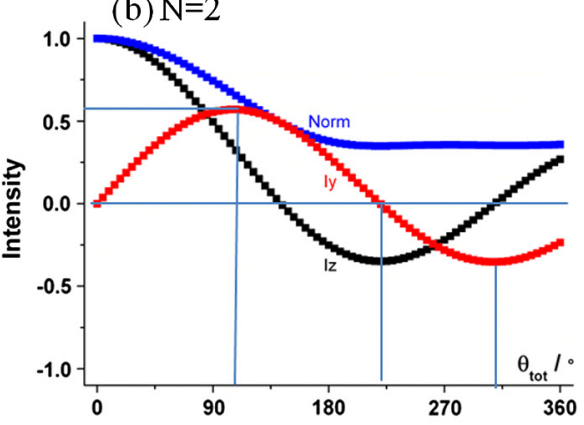

(d) $\mathrm{N}=4$

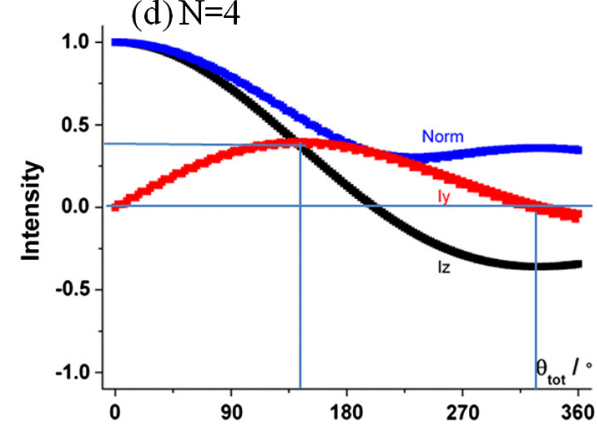

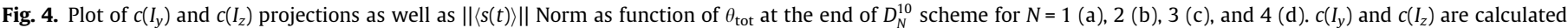
according to Eq. (2) and $\|\langle s(t)\rangle\|$ according to Eq. (3). $B_{0}=18.8 \mathrm{~T}, v_{R}=60 \mathrm{kHz}, v_{1}=29 \mathrm{kHz}$, and $\tau_{p}=\theta_{\text {tot }} /\left(3600 N v_{1}\right)$.

For $N=1$ (Fig. 4a), the norm remains close to unity, and the $y$ and $z$ components follow quasi-ideal sine and cosine functions, respectively. This is consistent with a quasi-ideal rotation of the magnetization about the $-x$ axis. Maximal transverse magnetization is obtained for $\theta_{\mathrm{tot}}^{\mathrm{opt}} \approx 90^{\circ}$. It is exactly $\theta_{\mathrm{tot}}^{\mathrm{opt}}=90^{\circ}$ when the $r f$-amplitude is large, e.g. $v_{1}=227 \mathrm{kHz}$ (Fig. S2b). The nutation effect of $D_{1}^{10}$ sequence with $r f$ field strength as low as $v_{1}=29 \mathrm{kHz}$ is quasi-identical to that of an ideal rectangular pulse. These simulations indicate that all pulses of a $D_{1}^{K}$ train have a cumulative effect, which stems from their rotor-synchronization, as explained below. The first pulse with phase $-x$ rotates the magnetization in the $y z$ plane. Hence, at the end of this pulse, the components of magnetization vector along $x, y$ and $z$ axes are $0, \sin \left(\theta_{\text {tot }}\right)$ and $\cos \left(\theta_{\text {tot }}\right)$ with $\theta_{\text {tot }}=2 \pi v_{1} \tau_{p}$. The subsequent free evolution causes the dephasing of the single-quantum coherences. However, dephasing under anisotropic interaction with second-rank orientation dependence is refocused at the end of each rotor period and transverse magnetization points again in the $\mathrm{y}$ direction, which corresponds to the formation of a rotational echo [9]. At the top of the echo, a second pulse is applied which rotates again the magnetization in the $y z$ plane, decreasing the magnetization along $z$ axis and augmenting that along $y$ axis. The synchronization between the refocusing of anisotropic interaction by MAS and the nutation of the magnetization by the $r f$ pulses produces an effective rotation of the magnetization about the $-x$ axis and the thermal equilibrium density matrix is transformed by $D_{1}^{K}$ pulse train into $\cos \left(\theta_{\text {tot }}\right) I_{z}+\sin \left(\theta_{\text {tot }}\right) I_{y}$, where now $\theta_{\text {tot }}=2 \pi K v_{1} \tau_{p}$. This predicted evolution is consistent with the simulations displayed in Figs. 4a and S2b. As shown in Fig. S2a, this unitary transformation by $D_{1}^{K}$ scheme requires the perfect rotor-synchronization of the pulses. Indeed, in the absence of rotor-synchronization, the pulses are not applied at the top of the rotational echo and hence the trajectory of magnetization is crystallite-dependant and the norm of powder magnetization is not conserved.

The $D_{1}^{K}$ sequence can thus be used as an excitation, an inversion or a refocusing pulse. This has been previously shown on $\mathrm{CeF}_{3}$
(Fig. 6 in Ref. [18]). However, experimentally the oscillations of the nutation curve were damped by irreversible losses.

For $N>1$ (Fig. 4b-d), the norm decreases for increasing $\theta_{\text {tot }}$ and the evolutions of $c\left(I_{y}\right)$ and $c\left(I_{z}\right)$ projections deviate from sine and cosine functions. With increasing $N$ values, (i) these deviations increase, and the maximum in $y$ total magnetization, $c\left(I_{y}\right)^{\text {opt }}$, (ii) decreases and (iii) is observed for larger $\theta_{\text {tot }}$ values: $\left(N, \theta_{\mathrm{tot}}^{\mathrm{opt}}, c\left(I_{y}\right)^{\mathrm{opt}}\right)=\left(1,95^{\circ}, 0.95\right),\left(2,110^{\circ}, 0.54\right),\left(3,125^{\circ}, 0.48\right), \quad(4$, $\left.155^{\circ}, 0.39\right)$ for $v_{1}=29 \mathrm{kHz}$ and $\left(1,90^{\circ}, 1\right),\left(2,110^{\circ}, 0.55\right),\left(3,125^{\circ}\right.$, $0.49)$ and $\left(4,155^{\circ}, 0.41\right)$, for $v_{1}=227 \mathrm{kHz}$. Note also that for $D_{N}^{10}$ scheme with $N>1$, and contrary to $D_{1}^{10}$ sequence, the maximum in $y$ magnetization is not concomitant with the cancelation of the $z$ magnetization. These simulations with $D_{N}^{10}$ sequences $(N>1)$ indicate destructive interferences between the different pulse trains, which stem from the absence of rotor-synchronization between the $N$ pulse trains. For instance, in $D_{2}^{10}$ sequence, the two pulse trains are shifted by half rotor period and when the second pulse is applied, the anisotropic interactions are not refocused. Consequently, the direction of transverse magnetization differs between the crystallites and the second pulse does not cause the $x$ components of magnetization to rotate. Furthermore, the nutation of the $y$ component of the magnetization by the second pulse train interferes with the refocusing of the anisotropic interaction by MAS. Therefore, a portion of the magnetization dephased by anisotropic interactions cannot be refocused at the end of the rotor period and hence does not contribute to the powder magnetization. It has already been reported that a long $r f$ pulse can scramble the magnetization of the different crystallites from powder samples when the nuclei are subject to large anisotropic interactions [4043]. This scrambling results in an apparent saturation of the magnetization of a powder sample. This phenomenon has been used for hetero-nuclear distance measurement [40-46]. Fig. S1 shows the build-up of powder $y$ magnetization of $D_{N}^{10}$ sequences with $N>1$ over a broader interval of $\theta_{\text {tot }}$ values. These build-up curves behave as damped-sine functions and are similar to the nutation curves of a single rectangular pulse in the presence of inhomogeneous $r f$ 
field. This analogy confirms that during $D_{N}^{10}$ sequences with $N>1$, the nutation of $y$ magnetizations differs between the crystallites. Additional simulations confirm that the magnetization losses for $D_{N}^{10}$ sequences with $N>1$ do not stem from insufficient $r f$ field strength. Indeed, the nutation curves for $v_{1}=227 \mathrm{kHz}$ (Fig. S2) are almost identical to those of Fig. 4 simulated with $v_{1}=29 \mathrm{kHz}$. As a result, the $D_{N}^{K}$ sequences $(N>1)$ produce efficient excitation, but are poorly efficient for inversion or refocusing.

The total integral of the $D_{N}^{K}$ spectrum, i.e. the sum of the integral of all the bands, is proportional to $I_{\text {norm }}$ (Eq. (4)), which is proportional to $c\left(I_{y}\right)$. As the integral of a given observable band is roughly equal to the total integral divided by the number of visible bands in the spectra, $N B$, the maximum intensity of each band is ca. proportional to $I I_{\text {norm }} / N B$ if all bands have the same line-width. Furthermore, according to Eq. (5), $N B$ is ca. inversely proportional to $N$, provided $F W>2 N v_{R}$, and hence the maximum intensity of each band is ca. proportional to $N I_{\text {norm. }}$. This is observable in Fig. 2, where the relative $r f$ center-band intensities, $I_{\mathrm{CB}}\left(D_{N}^{14}\right) / I_{\mathrm{CB}}\left(D_{1}^{14}\right)$, and the $N I I_{\text {norm }}\left(D_{N}^{14}\right) / N I I_{\text {norm }}\left(D_{1}^{14}\right)$ ratios are close, being respectively equal to $(1.16,1.08),(1.34,1.26)$, and $(1.50,1.56)$, for $N=2,3$, and 4 , respectively. Therefore, the quantity $N I I_{\text {norm }}$ is a reliable estimator of the relative visible-band intensities between two $D_{N}^{K}$ spectra, provided they use $F W>2 N v_{R}$ and the same $K T_{R}$ value, i.e. the same line-width according to Eq. (6) [9].

Additional simulations were also performed to check the dependence of the intensity as function of the various parameters of $D_{N}^{K}$ sequence. Fig. $5 a-d$ shows the variation of $N I I_{\text {norm }}$ as function of $K$ and $\tau_{p}$ parameters for $D_{N}^{K}$ scheme with $N=1,2,3$ or 4 , with $v_{1}=29 \mathrm{kHz}$. For these $N$ values, the corresponding $D_{N}^{K}$ spectra exhibit several bands in Fig. $2, F W>2 N v_{R}$, and hence $N I_{\text {norm }}$ is a reliable estimator of the band intensity. In these figures, the contour levels are close to hyperbolae with equation $\tau_{p}=\theta_{\text {tot }} /\left(360 K N v_{1}\right)$ (Eq. (1)). This dependence is confirmed by changing the vertical coordinate from $\tau_{p}$ to $\theta_{\text {tot }}$ (see Fig. 5e-h). This new representation highlights that at given $N$ and $\theta_{\text {tot }}$ values, the $y$ magnetization does not depend on the $K$ value, at least when the pulses are shorter than $0.65 \mu \mathrm{s}$, a duration short enough to excite the whole powder pattern (see Eq. (9)). Additional simulations shown in Fig. S3 with $v_{1}=227 \mathrm{kHz}$ confirm that the nutation curves do not depend on $r f$ field strength, provided the condition given in Eq. (9) is satisfied. As a conclusion, the intensity of $D_{N}^{K}$ spectra only depends on $\theta_{\text {tot }}$ and $N$ values.

We further investigated by numerical simulations the variation of the intensity of $D_{N}^{K}$ spectrum for a powder as function of $N$ values up to 32. As expected, the evolution of magnetization versus $K$
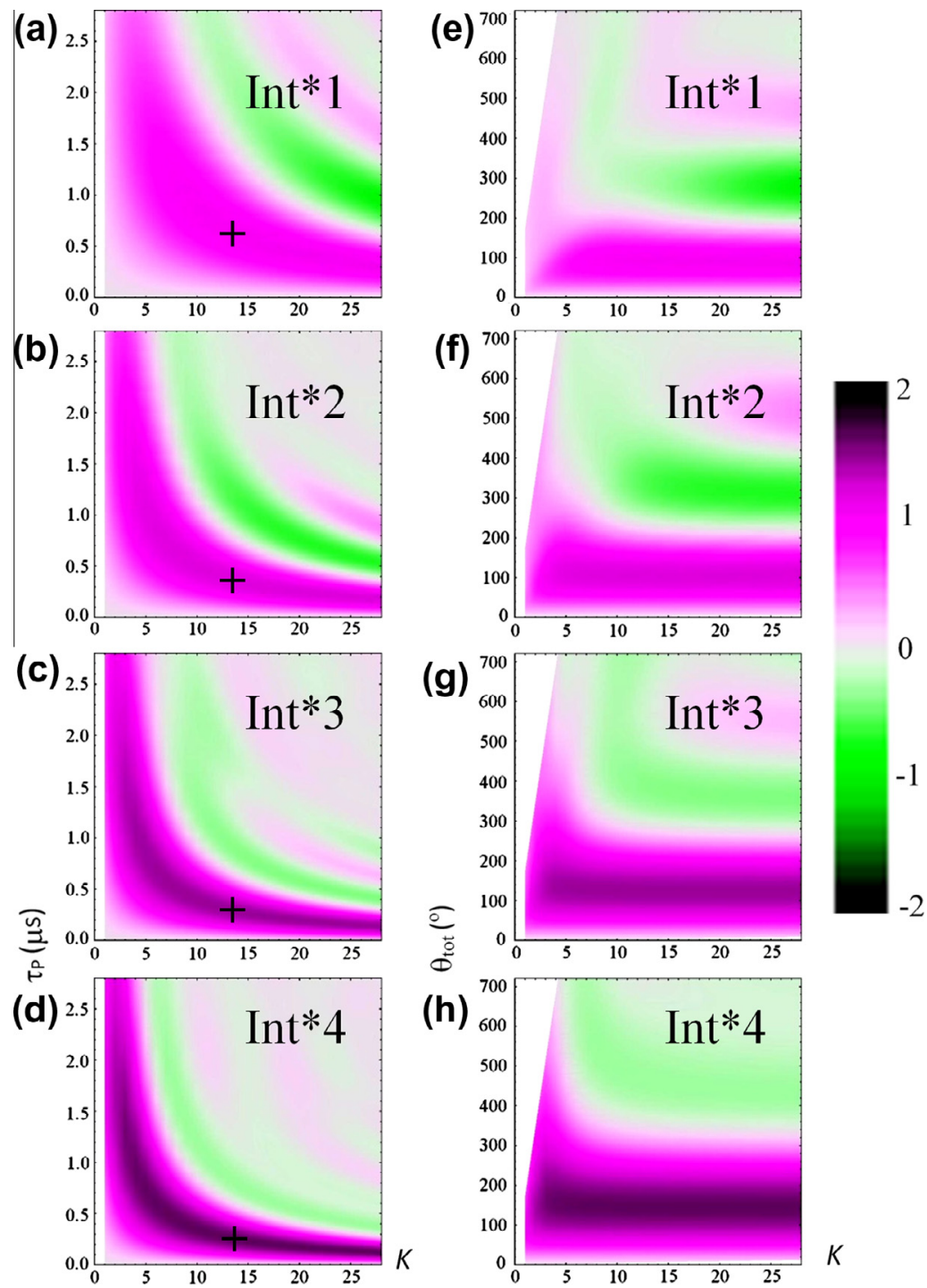

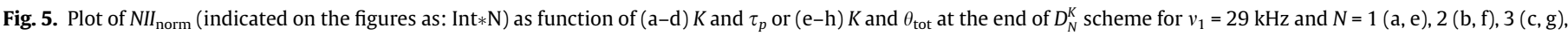
and $4(\mathrm{~d}, \mathrm{~h}) . I I_{\text {norm }}$ was calculated according to Eq. (4). The other simulation parameters are identical to those of Fig. 2. 

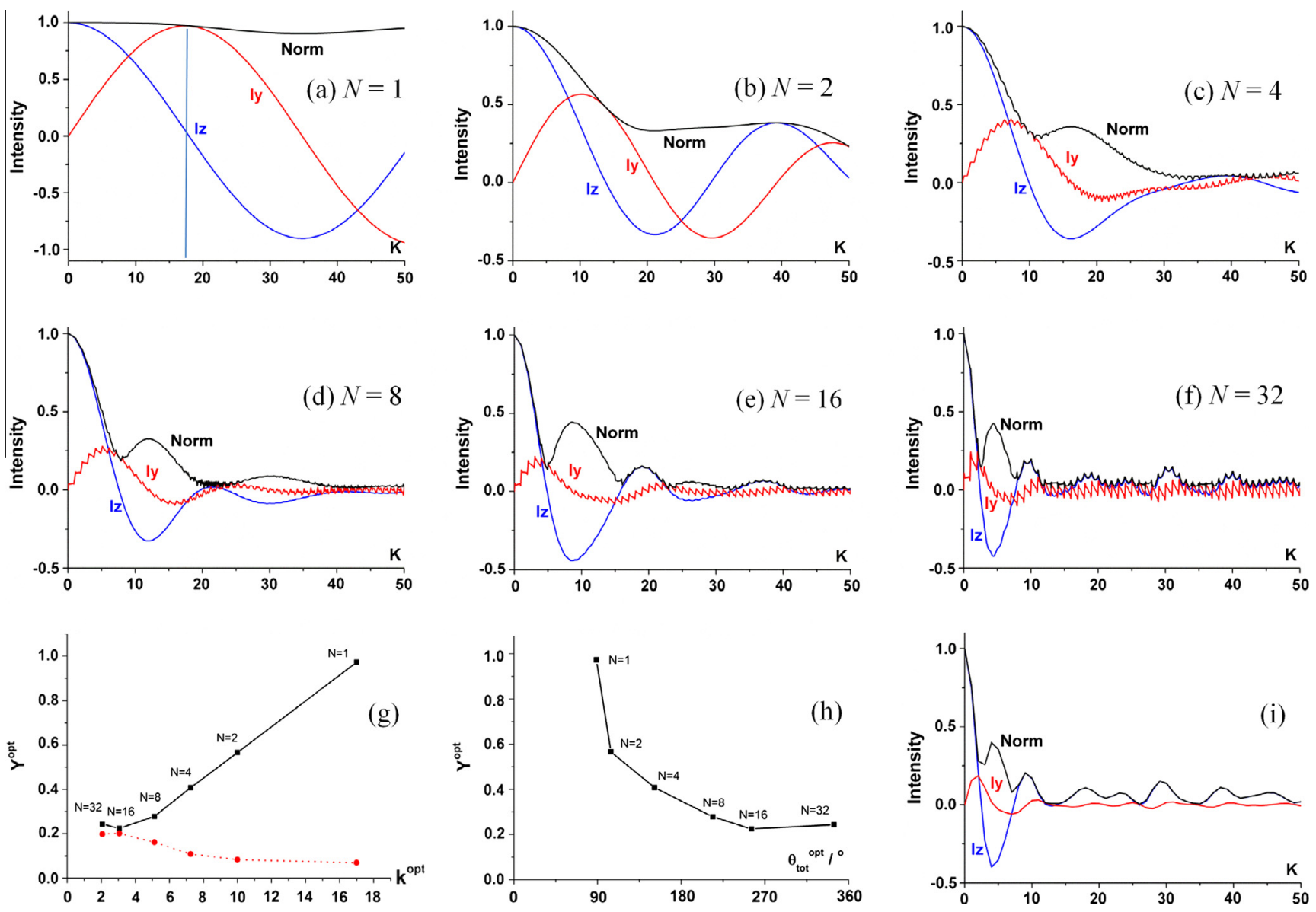

Fig. 6. (a-f) Plot of $c\left(I_{y}\right)$ and $c\left(I_{z}\right)$, as well as $\|\langle s(t)\rangle\|$ Norm as function of $K$ at the end of $D_{N}^{K}$ scheme for $N=1$ (a), 2 (b), 4 (c), $8(\mathrm{~d}), 16$ (e), 32 (f); with $\tau_{p}=0.5 \mu$ s and $v_{1}=29 \mathrm{kHz}$. Plot of $c\left(I_{y}\right)^{\text {opt }}$ as function of $K^{\text {opt }}(\mathrm{g})$ and $\theta_{\text {tot }}^{\text {opt }}(\mathrm{h})$. In $(\mathrm{g})$, the value of the center-band intensity is also indicated in red versus $N$ for $K^{\text {opt. }}$. (i) Plot of $c\left(I_{y}\right), c\left(I_{z}\right)$, and $\|\langle s(\mathrm{t})\rangle\|$ versus $K$ after one rectangular resonant pulse lasting $K T_{R}$. The other simulation parameters are identical to those of Fig. 2. For all sequences, $c\left(I_{x}\right)$ is zero. (For interpretation of the references to color in this figure legend, the reader is referred to the web version of this article.)

during $D_{N}^{K}$ sequence converges toward that observed with a single continuous rectangular pulse, as the duty cycle, $N \tau_{p} / T_{R}$, approaches 1 (compare Fig. $6 \mathrm{f}$ and i). Furthermore, for increasing $N$ value, the maximum in $y$ magnetization, $c\left(I_{y}\right)^{\text {opt }}$, which is obtained for smaller $K^{\text {opt }}$ and larger $\theta_{\text {tot }}^{\text {opt }}$ values (see Fig. $6 \mathrm{~g}$ and $\mathrm{h}$ ), decreases as already observed in Fig. 4. This decrease stems from the scrambling of the magnetization of the different crystallites by the $N$ pulse trains of $D_{N}^{K}$ sequence. However, it must be noted that for increasing $N$ value, $c\left(I_{y}\right)^{\text {opt }}$ decreases slower than $1 / N$ and hence that the intensity of each observable band, ca. $c\left(I_{y}\right)^{\text {opt }} / N B$, increases (see Fig. $6 \mathrm{~g}$ ) until the $D_{N}^{K}$ spectrum only exhibits a single band at the carrier frequency, i.e. for $N \geqslant F W /\left(2 v_{R}\right)$ (see Eq. (5)). Therefore, in direct excitation using $D_{N}^{K}$ sequences, optimal sensitivity $(\mathrm{S} / \mathrm{N})$ is achieved for $N \approx F W /\left(2 v_{R}\right) \approx 11$, in agreement with the results displayed in Fig. 6 g. $D_{N}^{K}$ spectra with $N=16$ and 32 exhibit a single center band. Similarly a resonant rectangular resonant pulse yields maximal $y$ magnetization for $\tau_{p} \approx 2 K T_{R}$ (see Fig. 6i) and according to Eq. (8), the optimized resonant rectangular pulse has a $\mathrm{FWE}_{0.5} \approx 0.675 v_{R}$ and hence is only able to excite the center band. Furthermore, the small $K^{\text {opt }}$ values of $D_{N}^{K}$ sequences associated with large $N$ values, i.e. their short lengths, lead to further advantages in terms of (i) sensitivity when additional coherent or incoherent mechanisms decrease the magnetization during the pulse train, and (ii) broader band-width to excite several different species.

According to the above simulations, the $D_{N}^{K}$ sequence used for the direct excitation of wide spectra can thus be optimized in the following way. First, highest possible spinning speed, $v_{R}$, is desirable since it decreases the length of $D_{N}^{K}$ sequences, which reduces the losses and broaden the $r f$ spikelets, hence improving the robustness to offset. Moreover, even with ultra fast spinning speed, wide spectra exhibit a sufficient number of spinning sidebands $\left(F W \gg v_{R}\right)$ for a reliable determination of the CSA parameters. Second, high $v_{1}$ amplitude delivered by probes with small diameter rotor and high MAS frequency allows decreasing the length of $D_{N}^{K}$ sequences, which also reduces the losses and broadens the $r f$ spikelets, in the same way as fast spinning speed. However, we have demonstrated experimentally that $D_{N}^{K}$ scheme with $r f$ amplitude as low as $v_{1}=29 \mathrm{kHz}$ can excite ${ }^{19} \mathrm{~F}$ spectra of ca. $1.3 \mathrm{MHz}$ wideness [9]. Third, according to Eq. (9), the pulse length can be chosen as $\tau_{p} \approx \max \left\{0.85 / F W, 0.85 / F W^{\text {probe }}\right\}$, in order to excite the full spectral width or at least the probe detection band-with. Indeed, shorter $\tau_{p}$ values are not recommended as, for fixed $v_{1}$ value, they lengthen the DANTE sequence, hence increasing the losses. Fourth, an optimal $\mathrm{S} / \mathrm{N}$ is achieved for $N \approx F W /\left(2 v_{R}\right)$, but such condition only excites the center band. Therefore, large $N$ values can only be used to determine the isotropic shifts of the different species (if they are sufficiently close to the carrier frequency), but not the CSA which requires smaller $N$ values. Fifth, the optimal number of rotor periods is given by

$K^{\mathrm{opt}}=\theta_{\mathrm{tot}}^{\mathrm{opt}}(N) /\left(360 N v_{1} \tau_{p}\right)$,

where the optimal flip angle, $\theta_{\mathrm{tot}}^{\mathrm{opt}}$, depends on $N$ value (see Fig. $6 \mathrm{~h}$ ). Sixth, when the spinning sidebands manifolds of chemically distinct sites with different isotropic chemical shifts must be excited by a single $D_{N}^{K}$ scheme, the length of the DANTE sequence must fulfill the relation $K<K^{\text {offset }}=0.85 v_{R} /\left[\Delta v_{\text {iso }}, \bmod \left(N v_{R}\right)\right]$ (see Eq. (7)), 


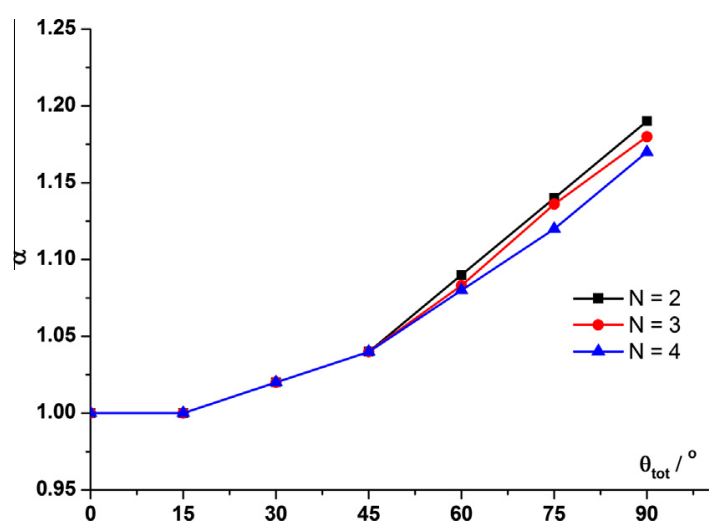

Fig. 7. Plot of $\alpha$ scaling factor as function of $\theta_{\text {tot }}$ angle for $v_{1}=29 \mathrm{kHz}$ and $N=2,3$ and 4 . The $\alpha$ values are determined by comparison between the simulated 1D spectra excited by $D_{N}^{5}$ sequence and $\sum_{N}^{5}$ sum of the $N$ individual trains of $D_{N}^{5}$ sequence (see Figs. S4 and S5). The other simulation parameters are identical to those of Fig. 2.

where $\Delta v_{\text {iso }}$ is the total spread of the different species. Therefore, when $K^{\text {offset }}<K^{\text {opt }}$, a choice has to be made between sensitivity and robustness to offset. The two options are then either the acquisition of a single $D_{N}^{K}$ spectrum with $K=K^{\text {offset }}$ and decreased efficiency, or the successive acquisition of the distinct spinning sidebands patterns with optimal efficiency $\left(K=K^{\mathrm{opt}}\right)$. The choice between these two options depends on the longitudinal relaxation times and the difference in isotropic shifts.

\subsection{Interferences between the N pulse trains of interleaved DANTE}

Up to now, mainly the total $y$ magnetization at the end of $D_{N}^{K}$ scheme, $c\left(I_{y}\right)$, has been considered. The decrease in $c\left(I_{y}\right)^{\text {opt }}$ magnetization for increasing $N$ value suggests interferences between the $N$ interleaved time-shifted pulse trains, which compose a $D_{N}^{K}$ sequence. However, the most relevant tool to analyze these interferences is based on the intensities of the spinning sidebands. To perform in details this examination we have compared the simulated spectra either excited by $D_{N}^{K}$ sequence or the sum $\left(\sum_{N}^{K}\right)$ of the $N$ spectra excited by the individual time-shifted $D_{1}^{K}$ pulse trains composing the $D_{N}^{K}$ sequence. In both cases, the transverse magnetization was recorded with the same time origin, the beginning of the first pulse of $D_{N}^{K}$ sequence. As shown in Figs. S4-S7, the $D_{N}^{5}$ and $\sum_{N}^{5}$ spectra exhibit identical envelopes but the intensities of all bands in $D_{N}^{5}$ spectra are uniformly scaled down by a factor $\alpha \geqslant 1$. Fig. 7 shows the variation of $\alpha$ as function of $\theta_{\text {tot }}$ for $D_{N}^{5}$ sequence with $N=2,3$ or 4 and $v_{1}=29 \mathrm{kHz}$. For $\theta_{\text {tot }} \leqslant 15^{\circ}, \alpha=1.0$ : the response to $D_{N}^{K}$ sequences is the sum of the responses to the individual pulse trains (see Fig. S5). For these small $\theta_{\text {tot }}$ flip angles, the excitation is in the linear regime and the $N$ pulse trains can be considered as independent. For $\theta_{\text {tot }}>15^{\circ}, \alpha>1$ and its value increases for increasing $\theta_{\text {tot }}$ angle. For instance, for $\theta_{\text {tot }}=90^{\circ}$, the intensities of $D_{N}^{5}$ spectra is about $20 \%$ lower than the sum of the individual spectra (see Fig. S4). These results indicate that: (i) the $N$ pulse trains of $D_{N}^{K}$ sequence interfere and (ii) the response to $D_{N}^{K}$ sequence is in the non-linear regime when $\theta_{\text {tot }}>15^{\circ}$. Moreover, additional simulations (see Figs. S6 and S7) show that for a given $\theta_{\text {tot }}$ flip angle, the $\alpha$ value is weakly dependent on the $r f$ field strength, and that its variations are limited to ca. $5 \%$.

\section{Simulation results for ${ }^{14} \mathrm{~N}$}

\subsection{Simulation of $D_{N}^{K}{ }^{14} N 1 D$ MAS NMR spectra}

Fig. 8 shows simulated 1D MAS ${ }^{14} \mathrm{~N}$ spectra with $C_{Q}=1.18 \mathrm{MHz}$ and $\eta_{Q}=0.5$, excited by one rectangular pulse or $D_{N}^{5}$ sequences, (a)

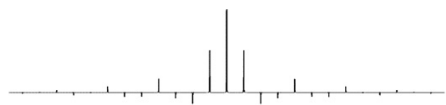

(b)

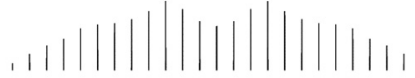

(c)

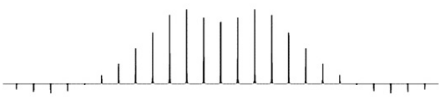
$\alpha=1.19$

(d)

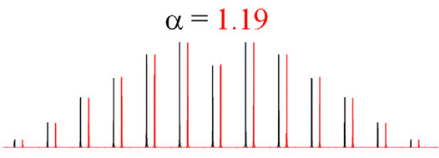
$\alpha=1.16$

(e)

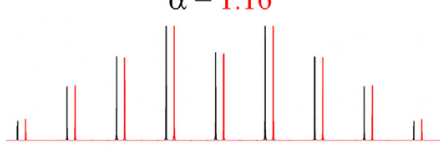
$\alpha=1.15$

(f)

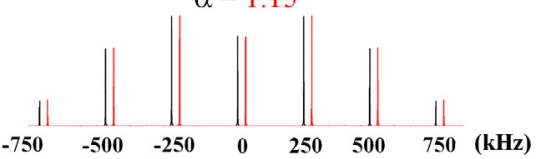

Fig. 8. Simulated $1 \mathrm{D}{ }^{14} \mathrm{~N}$ NMR spectra, represented over $\pm 800 \mathrm{kHz}$, and excited by: (a) one rectangular and resonant $90^{\circ}$ pulse lasting $10 \mathrm{~ms}$, (b) an ideal $90^{\circ}$ pulse, (cf) $D_{N}^{5}$ sequences (in black) with $N=1$ (c), 2 (d), 3 (e), and 4 (f). In (d-f), are also displayed in red the sum, $\sum_{N}^{5}$, of the $N$ individual trains of $D_{N}^{5}$ sequence. These spectra are simulated for an isolated ${ }^{14} \mathrm{~N}$ site subject to 1 st-order quadrupole interaction with $C_{Q}=1.18 \mathrm{MHz}, \eta_{Q}=0.5, B_{0}=18.8 \mathrm{~T}, v_{R}=62.5 \mathrm{kHz}, v_{1}=25 \mathrm{kHz}$, $\theta_{\text {tot }}=90^{\circ}$, and $\left(N, \tau_{p}(\mu \mathrm{s})\right)=(1,2)(\mathrm{c}),(2,1)(\mathrm{d}),(3,0.67)(\mathrm{e})$ and $(4,0.5)(\mathrm{f})$. The $D_{N}^{5}$ spectra are shifted to the left in (d-f) and scaled by the factor $\alpha$ indicated above the spectra. It must be noted that spectra calculated with 2nd-order quadrupole interaction are very similar to those presented here, owing to the moderate $C_{Q}$ and the large $B_{0}$ values. (For interpretation of the references to color in this figure legend, the reader is referred to the web version of this article.)

with $v_{1}=25 \mathrm{kHz}$. In Fig. 8, contrary to Fig. 2, the $\tau_{p}$ value is not optimized, but calculated so as to obtain $\theta_{\text {tot }}=90^{\circ}$. As shown in Fig. $8 \mathrm{~b}$, the spectrum excited by an ideal $90^{\circ}$ pulse spans a frequency range of $F W \approx 1.7 \mathrm{MHz}$. However, as shown in Fig. 8a, a resonant rectangular pulse of $10 \mu$ s excites sidebands across a width limited to ca. $120 \mathrm{kHz}$, in agreement with Eq. (8). A $D_{1}^{5}$ sequence with $\tau_{p}=2 \mu$ s is more broadband (see Fig. $8 \mathrm{c}$ ) and excites a frequency width of ca. $600 \mathrm{kHz}$, which is consistent with the $F W E_{0.5}$ value given by Eq. (8). As $F W$ exceeds $600 \mathrm{kHz}$, shorter pulses are thus required and according to Eq. (9), $\tau_{p} \leqslant 0.5 \mu$ s is essential to excite the full spectrum. In Fig. $8, v_{1}, K$ and $\theta_{\text {tot }}$ parameters are constant and hence $D_{N}^{5}$ sequences with larger $N$ values correspond to shorter pulse lengths. For instance, $\tau_{p}=0.5 \mu$ s for $D_{4}^{5}$ scheme and this sequence allows a uniform excitation of the full spectrum, as shown by comparison of Fig. $8 \mathrm{~b}$, e and f. $D_{1}^{20}$ scheme with $\tau_{p}=0.5 \mu \mathrm{s}$ and $\theta_{\text {tot }}=90^{\circ}$ also achieves broadband excitation of ${ }^{14} \mathrm{~N}$ spectrum (not shown).

Furthermore, similarly to those of ${ }^{19} \mathrm{~F}, D_{N}^{K}{ }^{14} \mathrm{~N}$ spectra exhibit envelopes that are identical to the sum of the $N$ spectra excited by the individual pulse trains composing the $D_{N}^{K}$ sequence. However, the intensity of $D_{N}^{K}$ spectra is again scaled down by $\alpha$ with respect to that of the sum spectra, $\sum_{N}^{K}$. These $\alpha$ factors are similar to those determined for ${ }^{19} \mathrm{~F} D_{N}^{K}$ spectra (see Fig. S4) and they confirm the interference between the $N$ pulse trains of $D_{N}^{K}$ sequence.

\subsection{Nutation curves of $D_{N}^{K}$ sequences}

Fig. 9 shows the nutation curves of $D_{N}^{K}$ sequences for ${ }^{14} \mathrm{~N}$ nucleus with $C_{Q}=1.18 \mathrm{MHz}$, i.e. the variation of $\mathrm{Nc}\left(\mathrm{I}^{-}\right)$as function of $\tau_{p}$ and $K$ parameters for $N=1,2,3$ or 4 . 
(a)

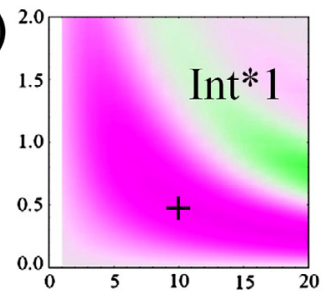

(b)
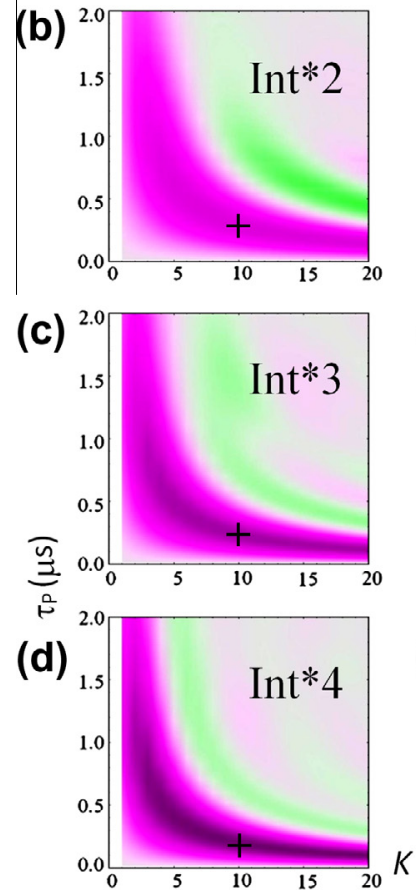

(e)

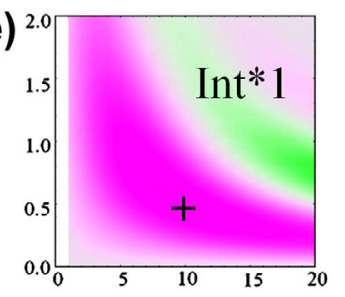

(f)

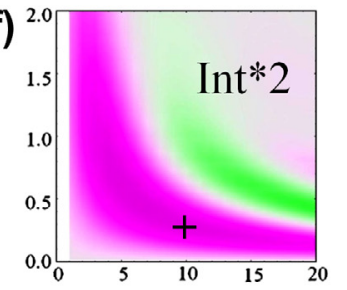

(g)

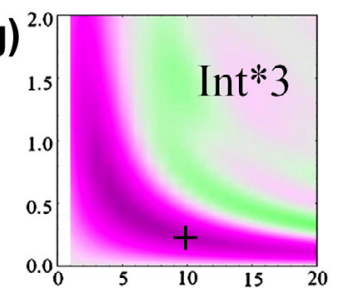

(h)

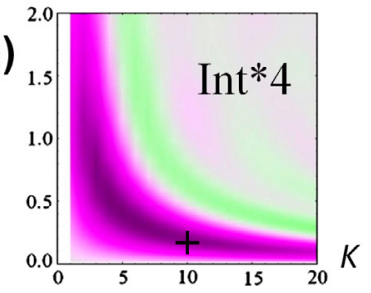

(i)

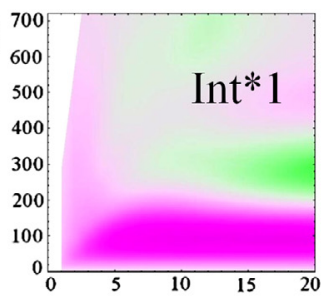

(j)

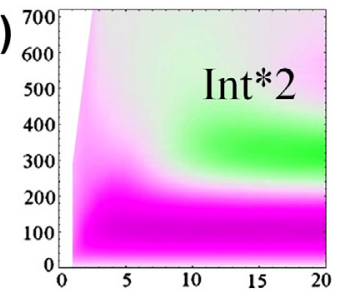

(k)

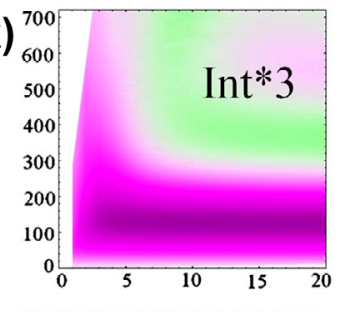

(I)

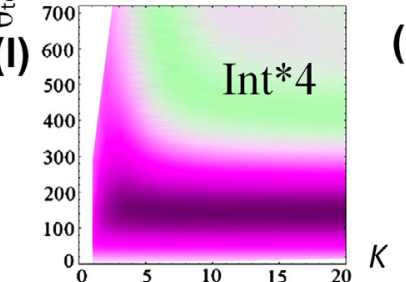

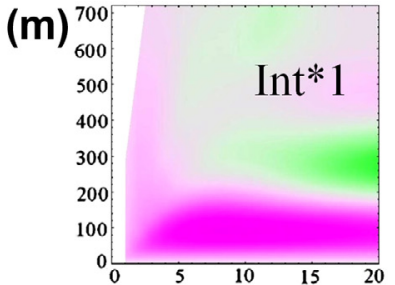

(n)

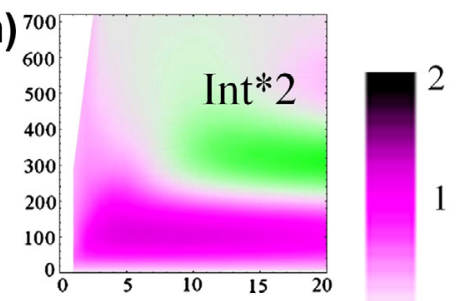

(o)
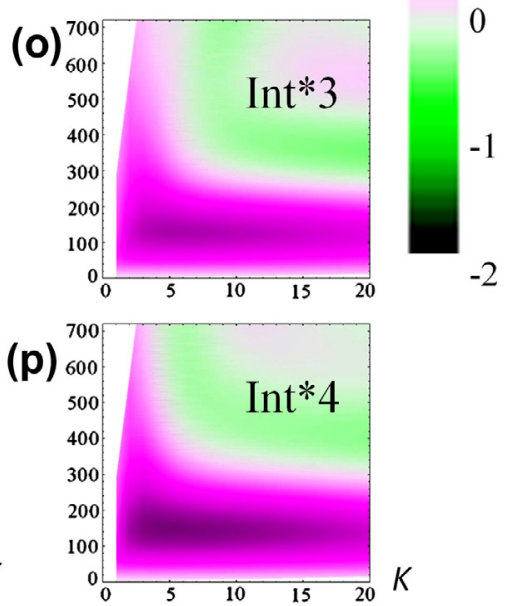

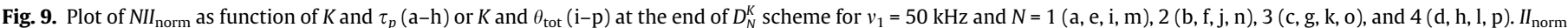

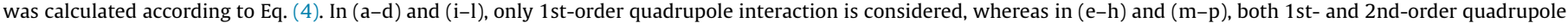

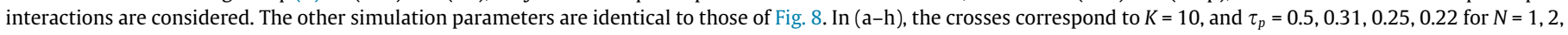
3,4 , respectively.

Simulations considering only the 1st-order quadrupolar terms are similar to those for ${ }^{19} \mathrm{~F}$ nucleus (compare Fig. 9a-d, i-l with Fig. 5). Indeed, for a given $\theta_{\text {tot }}$ angle, the $N c\left(I^{-}\right)$intensity does not depend on the $K$ parameter, provided $\tau_{p}$ is short enough to excite the whole powder pattern (see Fig. 9i-1). Moreover, similarly to the ${ }^{19} \mathrm{~F}$ simulations, $\theta_{\text {tot }}^{\text {opt }}$ angle for ${ }^{14} \mathrm{~N} D_{N}^{K}$ spectra (i) increases with $N$ value, confirming the interference between the $N$ pulse trains, and (ii) does not depend on the $r f$ field strength (compare Figs. 9 and S8).

Simulations were also performed in the presence of both firstand second-order quadrupole interactions. The second contribution contains terms with zero-, second- and fourth-rank orientation dependences, whereas the first one has purely a second-rank orientation dependence [37]. The terms with second-rank orientation dependence are refocused by MAS, whereas the other terms are not. Therefore, the dephasing produced by second-order quadrupole interaction is not refocused by MAS, which leads to intensity decrease for increasing length, $K T_{R}$, of $D_{N}^{K}$ sequences. However, this decrease is small in this case of weak $C_{Q}$ value and high $B_{0}$ field, and Fig. 9a-d are not very different from Fig. 9e-h. According to Eq. (10), the $K^{\text {opt }}$ value, and hence the optimal $D_{N}^{K}$ length, are inversely proportional to $v_{1}$ for a given $\tau_{p}$ length. Therefore, high $v_{1} r f$-field limits the dephasing due to second-order quadrupolar interaction (compare Figs. 9 and S8). Furthermore, as the second-order terms are proportional to $C_{Q}^{2}\left(1+\eta_{Q}^{2} / 3\right) / B_{0}$, the related loss of coherence increases at lower $B_{0}$ field and for ${ }^{14} \mathrm{~N}$ nuclei subject to larger quadrupolar interaction. This effect is exemplified in Fig. 10 showing the same results as in Fig. 9, but with a doubled quadrupolar interaction $\left(C_{Q}=2.36 \mathrm{MHz}\right)$, and hence a quadrupled second-order quadrupole interaction. In Fig. 10, second-order quadrupole interaction results in more pronounced intensity decrease for increasing $K$ value than in Fig. 9.

\subsection{Orientational selectivity of $D_{N}^{K}$ sequences}

The orientational selectivity of $D_{1}^{K}$ sequences was investigated by simulating $I I_{\text {norm }}$ as function of the $\left\{\alpha_{P L}, \beta_{P L}\right\}$ Euler angles describing the orientation of the efg Principal-axes system into the Laboratory frame at the time of the first rotational echo of the FID. The $I I_{\text {norm }}$ values were averaged using three $\gamma_{P L}$ angles $(0$, 120 and $240^{\circ}$ ) since crystallites with identical $\left\{\alpha_{P L}, \beta_{P L}\right\}$ angles but different $\gamma_{P L}$ angles have different orientations with respect to the rotor axis and hence different motions during the $D_{1}^{K}$ sequences. The numbers $\left\{I_{\text {norm }}, \alpha_{P L}, \beta_{P L}\right\}$ were plotted in spherical coordinates with a radial distance $I I_{\text {norm }}$, a polar angle, $\beta_{P L}$, and an azimuthal angle, $\alpha_{P L}$ (see Figs. 11 and 12). Note that contrary to the doughnut representation shown in Ref. [20], $I I_{\text {norm }}$ was not weighted by $\sin \left(\beta_{P L}\right)$, which is the probability distribution of crystallite orientations in a powder.

The selectivity of $D_{1}^{11}$ sequence was first investigated for ${ }^{14} \mathrm{~N}$ nucleus subject to a moderate quadrupole interaction $\left(C_{Q}=1.18\right.$ $\mathrm{MHz}$ ). For $v_{1}=50 \mathrm{kHz}$, the condition of Eq. (9) is valid and all crystallites are excited by the short $r f$ pulses with $\tau_{p}=454 \mathrm{~ns}$, even the crystallites with $\beta_{P L}=0^{\circ}$, which are then subject to the larger quadrupolar splitting of $\Delta v_{Q}=3 C_{Q} / 2$. Therefore, the excitation by $D_{1}^{11}$ sequence is equivalent to that by an ideal $90^{\circ}$ pulse, $I I_{\text {norm }}=1$ for 
(a)

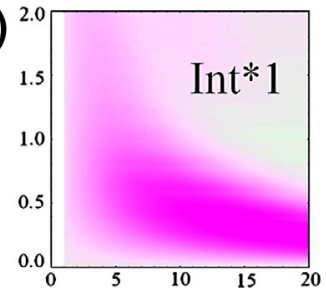

(b)
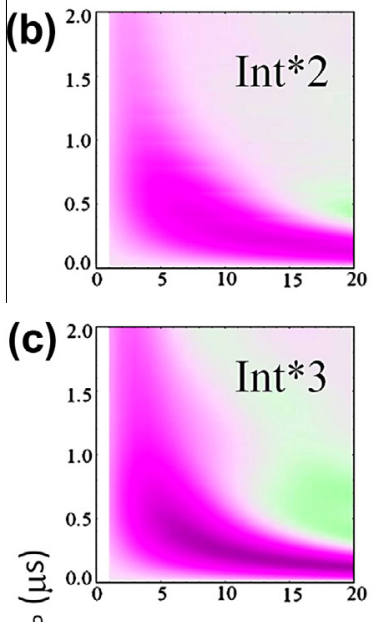

(d)

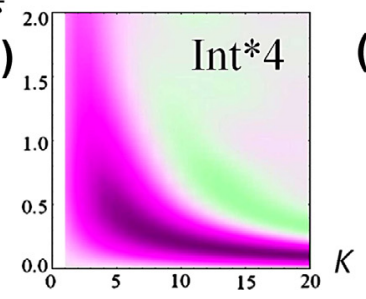

(e)

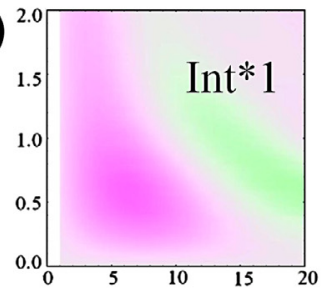

(f)

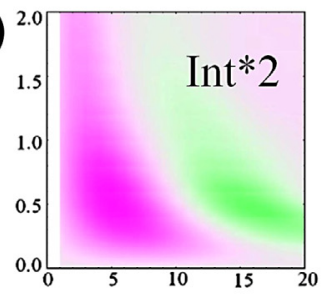

(g)

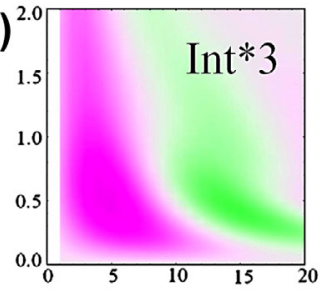

(h)

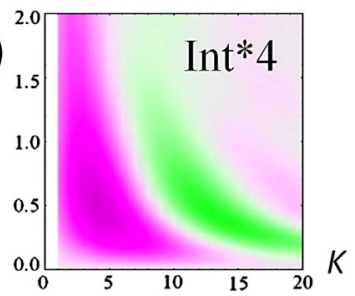

(i)

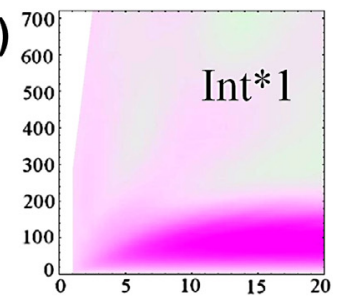

(j) 700

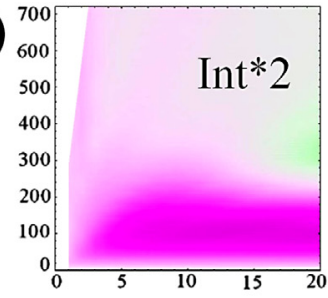

(k)

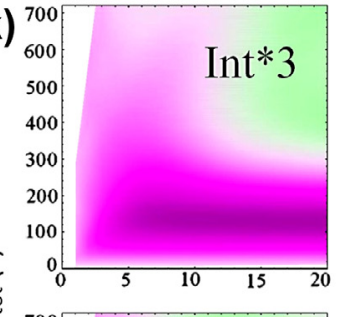

(I)

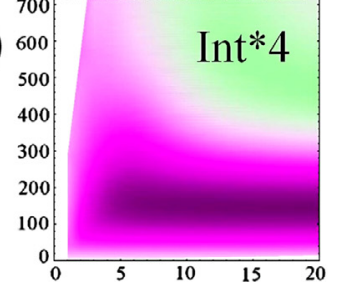

(m)

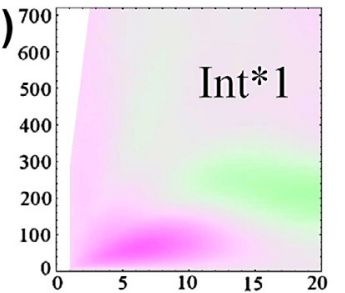

(n)

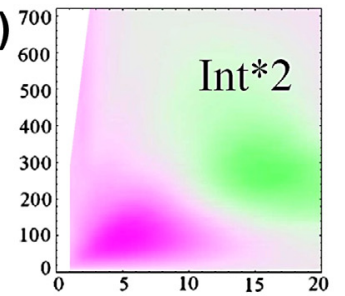

(o)

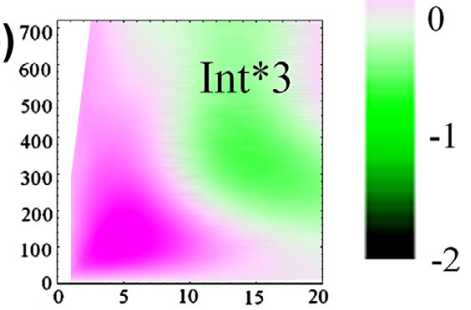

(p)

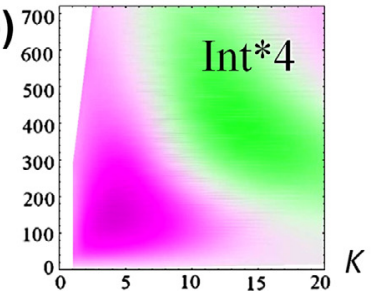

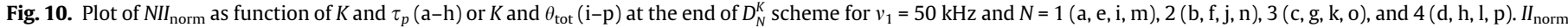

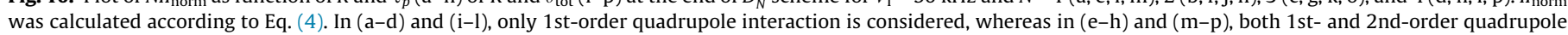
interactions are considered. $C_{Q}=2.36 \mathrm{MHz}$, the other simulation parameters are identical to those of Fig. 9 .

all $\left\{\alpha_{P L}, \beta_{P L}\right\}$ orientations, and the surface representing the orientational selectivity is nearly a sphere (see Fig. 11a). $D_{1}^{11}$ sequences with smaller $r f$ fields, and hence longer pulses: $\tau_{p}>500 \mathrm{~ns}$, are not able to excite the crystallites subject to larger quadrupolar interaction. The expression of the quadrupolar splitting is [37]

$\Delta v_{Q}=2\left|v_{Q}\right|=\frac{3 C_{Q}}{4}\left|3 \cos ^{2}\left(\beta_{P L}\right)-1+\eta_{Q} \sin ^{2}\left(\beta_{P L}\right) \cos \left(2 \alpha_{P L}\right)\right|$.

where $v_{Q}$ is the quadrupole frequency. This splitting is equal to $3 C_{Q} / 2$ for $\beta_{P L}=0^{\circ}$ and it cancels for $\beta_{P L}$ angles close to the magic angle $54.7^{\circ}$. For $\eta_{Q}=0.5, \Delta v_{Q} \approx 0$ for $\beta_{P L}$ angles ranging from $49^{\circ}$ to $63^{\circ}$ and is equal to $9 C_{Q} / 8$ for $\left\{\alpha_{P L}, \beta_{P L}\right\}=\left\{90^{\circ}, 90^{\circ}\right\}$. In other words, the crystallites oriented at Euler angles $\beta_{P L}=0^{\circ}$ and $\left\{\alpha_{P L}, \beta_{P L}\right\}=\left\{90^{\circ}\right.$, $90^{\circ}$ \} are subject to large quadrupole interactions during the $r f$ pulses, whereas those oriented at $\beta_{P L} \approx 54.7^{\circ}$ or $180^{\circ}-54.7^{\circ}$ are subject to vanishing quadrupole interactions. Therefore, in Fig. $11 \mathrm{~b}$ and $\mathrm{c}, I I_{\mathrm{norm}}$ is small for $\beta_{P L}=0^{\circ}$ and $\left\{\alpha_{P L}, \beta_{P L}\right\}=\left\{90^{\circ}, 90^{\circ}\right\}$ and larger for $\beta_{P L} \approx 54.7^{\circ}$. For long pulses, $I I_{\text {norm }}$ is smaller than 1 even for $\beta_{P L} \approx 54.7^{\circ}$ since owing to MAS, the orientations of all crystallites during the pulses vary with respect to the laboratory frame. For instance, for $v_{R}=62.5 \mathrm{kHz}$, the rotor rotates by $20^{\circ}$ and $40^{\circ}$ during a pulse lasting $\tau_{p}=909 \mathrm{~ns}$ and $1818 \mathrm{~ns}$, respectively. As the second-order quadrupole interaction is proportional to $C_{Q}^{2} / B_{0}$, the similarity of Fig. 11c $\left(B_{0}=18.8 \mathrm{~T}\right)$ and Fig. $11 \mathrm{~d}\left(B_{0} \rightarrow \infty\right)$ confirms that second-order quadrupole dephasings are negligible during $D_{1}^{11}$ sequence for $C_{Q}=1.18 \mathrm{MHz}$ and $B_{0}=18.8 \mathrm{~T}$.
Conversely, for larger $C_{Q}$ values, there are significant losses of coherence due to second-order quadrupole interactions during $D_{1}^{11}$ sequence (compare Fig. $12 \mathrm{~d}$ and e) and $D_{1}^{11}$ sequence does not achieve uniform excitation of all crystallites, even for short pulses verifying the condition given by Eq. (9). For instance, in Fig. $12 \mathrm{~b}$ and $\mathrm{d}, \tau_{p}<0.85 / \mathrm{FW}=240 \mathrm{~ns}$ for $C_{Q}=2.36 \mathrm{MHz}$ and 120 ns for $C_{Q}=4.72 \mathrm{MHz}$, but there are crystallites with $I I_{\text {norm }}<1$ and the surfaces representing the orientational selectivity differs from a sphere. Note also that in the presence of de-coherence due to the second-order quadrupole interaction, $I I_{\text {norm }}$ differs between the orientations $\beta_{P L}$ and $180^{\circ}-\beta_{P L}$. A solution to limit the losses due to second-order quadrupole interaction consists in using shorter $K$ values and hence shorter $D_{1}^{K}$ sequences. However, in Fig. $12 \mathrm{f}$, the length of the pulses does not verify the condition of Eq. (9) and hence the $D_{1}^{3}$ sequence is only able to excite the orientations with $\beta_{P L} \approx 54.7^{\circ}$ or $180^{\circ}-54.7^{\circ}$, for which the first-order quadrupole interaction is small.

For $D_{N}^{K}$ sequences with $N \geqslant 2$, the crystallites have distinct orientations with respect to the laboratory frame during the $r f$ pulses of the $N$ trains and hence they are subject to distinct quadrupole interactions. Therefore, the dependence of $I I_{\text {norm }}$ with respect to $\left\{\alpha_{P L}, \beta_{P L}\right\}$ Euler angles is more complex and e.g. for $D_{2}^{11}$ sequence the surfaces of Fig. S9 exhibit many sharp peaks and they look like the coat of porcupines. The comparison between Fig. S9a and b shows that this complex dependence does not stem from the second-order quadrupole interaction. 
(a)

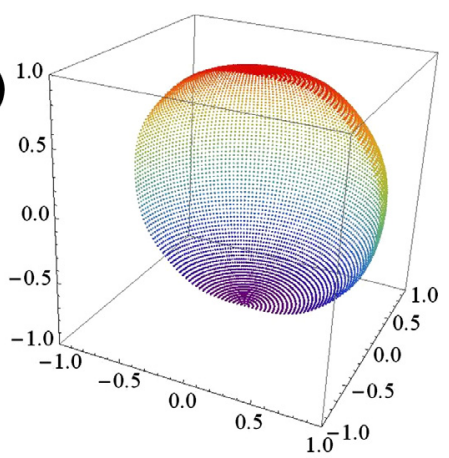

(c) 1.0

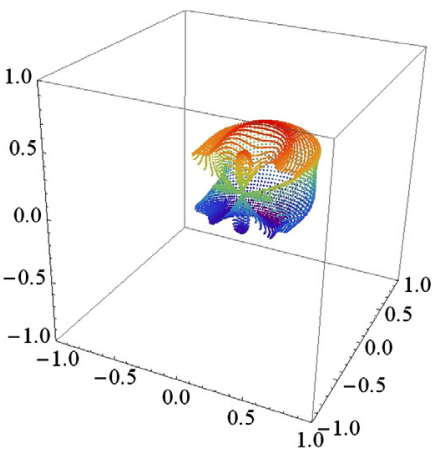

(b)
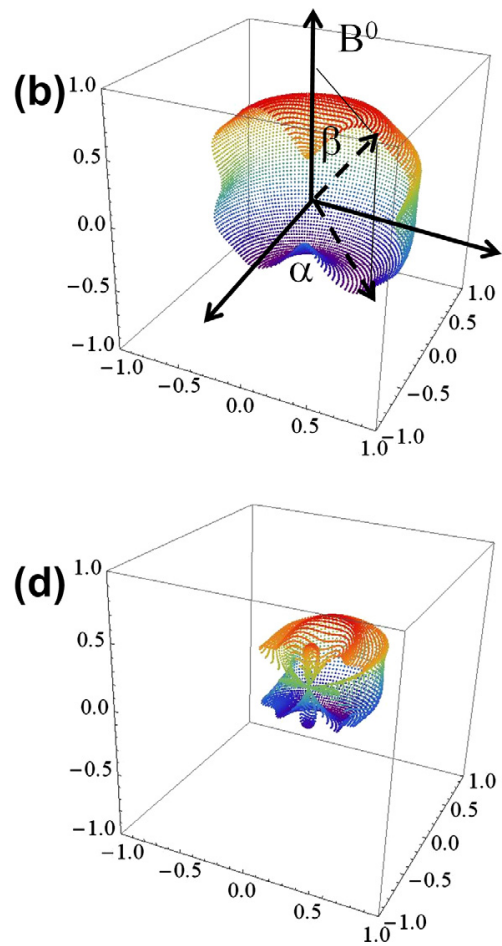

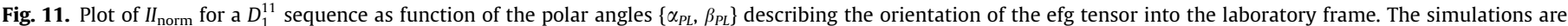

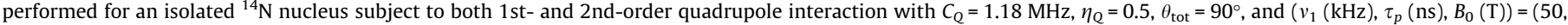
$454,18.8)$ (a), $(25,909,18.8)$ (b), $(12.5,1818,18.8)(c)$, and $(12.5,1818, \infty)(d)$.

(a)

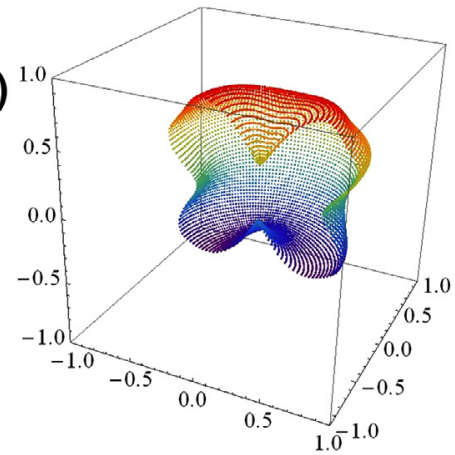

(d)

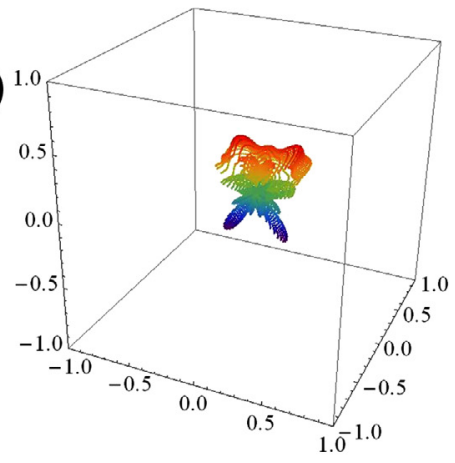

(b)

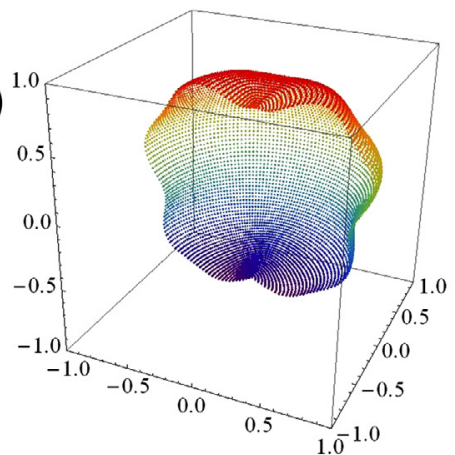

(e)

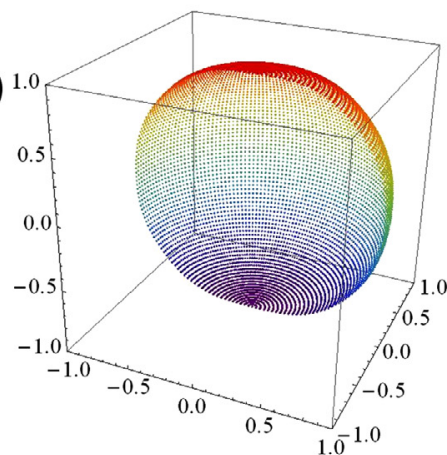

(c)

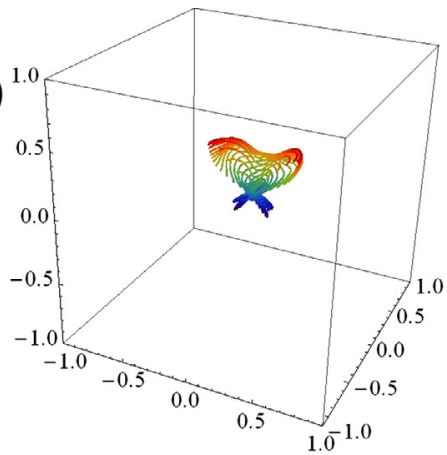

(f)

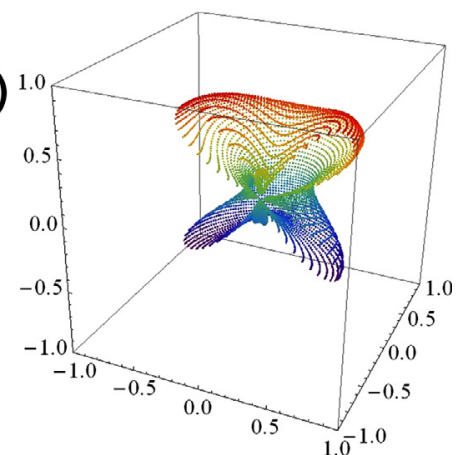

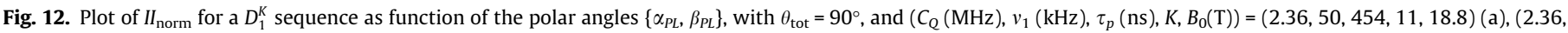

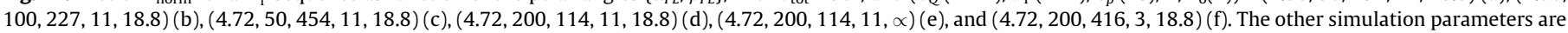
identical to those of Fig. 11. 


\section{Conclusions}

This article provides useful guidelines for the optimization of $D_{N}^{K}$ sequences used for the excitation of wide 1D spectra of spin-1/2 and spin-1 nuclei. Guidelines are also given for $D_{N}^{K}$ sequences used for refocusing or population inversion. Using numerical simulations, several properties of $D_{N}^{K}$ sequences have been analyzed, including the robustness to offset, the nutation curves, the interference between the pulse trains and the orientational selectivity.

Globally, the excitation profile has a comb shape, with $N v_{R}$ spacing between the bands from the carrier frequency, extending over a few MHz. We have shown that $r f$ pulses of $D_{N}^{K}$ scheme must be short enough to excite the minimum bandwidth value between the spectrum and the probe. High MAS frequency and high $r f$ field strength are required since they decrease the length of $D_{N}^{K}$ scheme, hence reducing the losses and improving the robustness to offset.

Depending on the length of the $D_{N}^{K}$ pulse train, i.e. the $K$ value, the selectivity can be tailored and the spinning sideband manifold of either all or only one species can thus be acquired.

An optimal sensitivity $(\mathrm{S} / \mathrm{N})$ is achieved for $N \approx F W /\left(2 v_{R}\right)$, but the $D_{N}^{K}$ spectra obtained under such conditions only contain the resonances close to the carrier frequency. Therefore, large $N$ values can only be used to determine the isotropic shifts if the differences between the carrier frequency and the isotropic chemical shifts are less than about $F W S_{0.5}$.

Short $D_{N}^{K}$ schemes are especially required in the presence of fast coherence losses due to homo-nuclear dipolar or second-order quadrupole interactions. The nutation curves of $D_{1}^{K}$ sequence are similar to that of an ideal resonant, rectangular pulse. In particular, this sequence allows a perfect inversion or refocusing of the longitudinal magnetization. Conversely, in interleaved DANTE sequences with $N \geqslant 2$, the interferences between the $N$ pulse trains produce coherence losses and the optimal cumulated excitation flip angle exceeds $90^{\circ}$. These sequences are poorly efficient for inversion or refocusing purposes. We have shown that the nutation during $D_{N}^{K}$ sequence depends on the cumulated flip angle during the $N K r f$ pulses. Furthermore, we have also shown that the orientational selectivity of interleaved DANTE sequences is more complicated than that of $D_{1}^{K}$ scheme.

\section{Acknowledgments}

This work was supported by: Swiss National Science Foundation (SNSF), Ecole Polytechnique Fédérale de Lausanne (EPFL), Swiss Commission for Technology and Innovation (CTI), Fédération de Recherche CNRS (FR-3050) Très Grands Equipements de RMN à Très Hauts Champs (TGIR RMN THC), French contract ANR-2010jcjc-0811-01. X.L., J.T., O.L., and J.P.A. are grateful for funding provided by Région Nord/Pas de Calais, European Union (FEDER), CNRS, French Ministry of Scientific Research, USTL, ENSCL, CortecNet and Bruker BIOSPIN.

\section{Appendix A. Supplementary material}

Supplementary data associated with this article can be found, in the online version, at http://dx.doi.org/10.1016/j.jmr.2013.09.003.

\section{References}

[1] R.W. Schurko, eMagRes (2011).

[2] N.P. Wickramasinghe, Y. Ishii, J. Magn. Reson. 181 (2006) 233.

[3] N.P. Wickramasinghe, M.a. Shaibat, C.R. Jones, L.B. Casabianca, A.C. de Dios, J.S Harwood, Y. Ishii, J. Chem. Phys. 128 (2008) 052210.

[4] A.J. Pell, R.J. Clément, C.P. Grey, L. Emsley, G. Pintacuda, J. Chem. Phys. 138 (2013) 114201.

[5] I. Hung, L. Zhou, F. Pourpoint, C.P. Grey, Z. Gan, J. Am. Chem. Soc. 134 (2012) 1898.

[6] G. Kervern, G. Pintacuda, L. Emsley, Chem. Phys. Lett. 435 (2007) 157.

[7] A.J. Pell, G. Kervern, L. Emsley, M. Deschamps, D. Massiot, P.J. Grandinetti, G. Pintacuda, J. Chem. Phys. 134 (2011) 024117.

[8] R.J. Clément, A.J. Pell, D.S. Middlemiss, F.C. Strobridge, J.K. Miller, M.S Whittingham, L. Emsley, C.P. Grey, G. Pintacuda, J. Am. Chem. Soc. 134 (2012) 17178 .

[9] D. Carnevale, V. Vitzthum, O. Lafon, J. Trébosc, J. Amoureux, G. Bodenhausen, Chem. Phys. Lett. 553 (2012) 68.

[10] M. Murakami, H. Arai, Y. Uchimoto, Z. Ogumi, J. Magn. Reson. 231 (2013) 66.

[11] D. Carnevale, a.J. Perez Linde, G. Bauer, G. Bodenhausen, Chem. Phys. Lett. 580 (2013) 172

[12] G. Kervern, G. Pintacuda, Y. Zhang, E. Oldfield, C. Roukoss, E. Kuntz, E. Herdtweck, J.-M. Basset, S. Cadars, A. Lesage, C. Copéret, L. Emsley, J. Am. Chem. Soc. 128 (2006) 13545.

[13] S. Cavadini, A. Lupulescu, S. Antonijevic, G. Bodenhausen, J. Am. Chem. Soc. 128 (2006) 7706.

[14] Z. Gan, J. Am. Chem. Soc. 128 (2006) 6040.

[15] Y. Nishiyama, Y. Endo, T. Nemoto, H. Utsumi, K. Yamauchi, K. Hioka, T. Asakura, J. Magn. Reson. 208 (2011) 44.

[16] T.J. Bastow, M.E. Smith, Solid State Nucl. Magn. Reson. 1 (1992) 165.

[17] D. Massiot, I. Farnan, N. Gautier, D. Trumeau, A. Trokiner, J.P. Coutures, Solid State Nucl. Magn. Reson. 4 (1995) 241.

[18] L.A. O'Dell, R.W. Schurko, Chem. Phys. Lett. 464 (2008) 97.

[19] L.A. O’Dell, R.W. Schurko, J. Am. Chem. Soc. 131 (2009) 6658.

[20] V. Vitzthum, M. a Caporini, S. Ulzega, J. Trébosc, O. Lafon, J.-P. Amoureux, G. Bodenhausen, J. Magn. Reson. 223 (2012) 228.

[21] V. Vitzthum, M.A. Caporini, S. Ulzega, G. Bodenhausen, J. Magn. Reson. 212 (2011) 234.

[22] T. Giavani, H. Bildsøe, J. Skibsted, H.J. Jakobsen, J. Magn. Reson. 166 (2004) 262.

[23] L.A. O’Dell, R.W. Schurko, K.J. Harris, J. Autschbach, C.I. Ratcliffe, J. Am. Chem. Soc. (2010) 527.

[24] A.L. Webber, S. Masiero, S. Pieraccini, J.C. Burley, A.S. Tatton, D. Iuga, T.N. Pham, G.P. Spada, S.P. Brown, J. Am. Chem. Soc. 133 (2011) 19777.

[25] Z. Gan, J. Magn. Reson. 184 (2007) 39.

[26] H.E. Rhodes, P.-K. Wang, T. Stokes, C.P. Slichter, J.H. Sinfelt, Phys. Rev. B 26 (1982) 3559.

[27] A.W. MacGregor, L.A. O’Dell, R.W. Schurko, J. Magn. Reson. 208 (2011) 103.

[28] G. Bodenhausen, R. Freeman, G.A. Morris, J. Magn. Reson. 23 (1976) 171.

[29] P. Caravatti, G. Bodenhausen, R.R. Ernst, J. Magn. Reson. 103 (1983) 88.

[30] D. Wei, U. Akbey, B. Paaske, H. Oschkinat, B. Reif, M. Bjerring, N.C. Nielsen, J. Phys. Chem. Lett. 2 (2011) 1289.

[31] M. Bak, J.T. Rasmussen, N.C. Nielsen, J. Magn. Reson. 147 (2000) 296.

[32] M. Alla, E. Lippmaa, Chem. Phys. Lett. 87 (1982) 30.

[33] A. Nayeem, J.P. Yesinowski, J. Chem. Phys. 89 (1988) 4600.

[34] A.R. Brough, C.P. Grey, C.M. Dobson, J. Am. Chem. Soc. 115 (1993) 7318.

[35] C.A. McDowell, A. Naito, D.L. Sastry, K. Takegoshi, J. Magn. Reson. 69 (1986) 283.

[36] A.S. Tatton, T.N. Pham, F.G. Vogt, D. Iuga, A.J. Edwards, S.P. Brown, CrystEngComm 14 (2012) 2654.

[37] S.E. Ashbrook, S. Wimperis, eMagRes (2009).

[38] C. Fernandez, M. Pruski, Top. Curr. Chem. 306 (2012) 119.

[39] M. Bak, N.C. Nielsen, J. Magn. Reson. 125 (1997) 132.

[40] K. Schmidt-Rohr, J.-D. Mao, J. Magn. Reson. 359 (2002) 403.

[41] X. Lu, O. Lafon, J. Trébosc, J.-P. Amoureux, J. Magn. Reson. 215 (2012) 34.

[42] E. Nimerovsky, A. Goldbourt, J. Magn. Reson. 225 (2012) 130.

[43] Z. Gan, Chem. Commun. (2006) 4712.

[44] L. Chen, Q. Wang, B. Hu, O. Lafon, J. Trébosc, F. Deng, J.-P. Amoureux, Phys. Chem. Chem. Phys. 12 (2010) 9395.

[45] L. Chen, X. Lu, Q. Wang, O. Lafon, J. Trébosc, F. Deng, J.-P. Amoureux, J. Magn. Reson. 206 (2010) 269.

[46] F. Pourpoint, J. Trébosc, R.M. Gauvin, Q. Wang, O. Lafon, F. Deng, J.-P. Amoureux, ChemPhysChem 13 (2012) 3605. 